\title{
Evolution of the eastward shift in the quasi-stationary minimum of the Antarctic total ozone column
}

\author{
Asen Grytsai ${ }^{1}$, Andrew Klekociuk ${ }^{2,3}$, Gennadi Milinevsky ${ }^{1,4}$, Oleksandr Evtushevsky ${ }^{1}$, and Kane Stone ${ }^{5,6, a}$ \\ ${ }^{1}$ Taras Shevchenko National University of Kyiv, 01601 Kyiv, Ukraine \\ ${ }^{2}$ Antarctica and the Global System Program, Australian Antarctic Division, Kingston, Tasmania 7050, Australia \\ ${ }^{3}$ Antarctic Climate and Ecosystems Cooperative Research Centre, Hobart, Tasmania 7000, Australia \\ ${ }^{4}$ Main Astronomical Observatory, National Academy of Sciences of Ukraine, 03143 Kyiv, Ukraine \\ ${ }^{5}$ School of Earth Sciences, University of Melbourne, Melbourne, Victoria 3010, Australia \\ ${ }^{6}$ ARC Centre of Excellence for Climate System Science, University of New South Wales, \\ Sydney, New South Wales 2052, Australia \\ a currently at: the Department of Earth, Atmospheric and Planetary Sciences, Massachusetts Institute of Technology, \\ Cambridge, Massachusetts 02139-4307, USA
}

Correspondence to: Gennadi Milinevsky (genmilinevsky@gmail.com)

Received: 19 June 2016 - Published in Atmos. Chem. Phys. Discuss.: 4 August 2016

Revised: 28 December 2016 - Accepted: 10 January 2017 - Published: 3 February 2017

\begin{abstract}
The quasi-stationary pattern of the Antarctic total ozone has changed during the last 4 decades, showing an eastward shift in the zonal ozone minimum. In this work, the association between the longitudinal shift of the zonal ozone minimum and changes in meteorological fields in austral spring (September-November) for 1979-2014 is analyzed using ERA-Interim and NCEP-NCAR reanalyses. Regressive, correlative and anomaly composite analyses are applied to reanalysis data. Patterns of the Southern Annular Mode and quasi-stationary zonal waves 1 and 3 in the meteorological fields show relationships with interannual variability in the longitude of the zonal ozone minimum. On decadal timescales, consistent longitudinal shifts of the zonal ozone minimum and zonal wave 3 pattern in the middle-troposphere temperature at the southern midlatitudes are shown. Attribution runs of the chemistry-climate version of the Australian Community Climate and Earth System Simulator (ACCESS-CCM) model suggest that long-term shifts of the zonal ozone minimum are separately contributed by changes in ozone-depleting substances and greenhouse gases. As is known, Antarctic ozone depletion in spring is strongly projected on the Southern Annular Mode in summer and impacts summertime surface climate across the Southern Hemisphere. The results of this study suggest that changes in zonal ozone asymmetry accompanying ozone depletion
\end{abstract}

could be associated with regional climate changes in the Southern Hemisphere in spring.

\section{Introduction}

The distribution of total column ozone over Antarctica is significantly non-uniform during austral spring, i.e., in September-November (Wirth, 1993; Malanca et al., 2005; Grytsai et al., 2007a; Agosta and Canziani, 2010, 2011). This has particularly been the case since the early 1980s due to the presence of ozone depletion associated with the ozone hole (Chubachi, 1984; Farman et al., 1985; Chubachi and Kajiwara, 1986; Stolarski et al., 1986; Solomon, 1999). The total ozone distribution predominantly characterizes the stratosphere layer, where a sharp ozone maximum is usually observed at altitudes of 15-25 km (Chubachi, 1984; Solomon et al., 2005). Therefore, strong variations in the total ozone distribution are due mainly to stratospheric chemistry and dynamics (Wirth, 1993; Gabriel et al., 2011). The ozone hole is located inside the polar stratospheric vortex, which is a cyclonic structure that impedes mixing between high-latitude and midlatitude air masses (Brasseur et al., 1997). The polar vortex is under the influence of large-scale planetary waves, which disturb the vortex edge region (Wirth, 1993; Quinta- 
nar and Mechoso, 1995) and the vortex location relative to the pole (Waugh and Randel, 1999). The scale of the waves in the zonal direction is typically characterized by zonal wave number, which is equal to the ratio of the corresponding great circle circumference at a given latitude to the wave length (Hirota and Hirooka, 1984; Hio and Yoden, 2004). The stationary part of the wave structure in the Southern Hemisphere ( $\mathrm{SH})$ spring stratosphere is mainly determined by a planetary wave with zonal number 1 (Hartmann et al., 1984; Quintanar and Mechoso, 1995; Ialongo et al., 2012), i.e., wave 1 . The role of planetary waves was especially important in the unusual SH stratospheric warming in 2002 (Varotsos, 2002; Allen et al., 2003; Hoppel et al., 2003). Both wave-1 and wave- 2 activity during austral winter and spring caused strong deceleration and warming of the stratospheric polar vortex, its anomalous splitting and ozone hole breakup in September 2002 (Varotsos, 2002; Nishii and Nakamura, 2004; Newman and Nash, 2005; Peters et al., 2007; Grassi et al., 2008; Peters and Vargin, 2015).

In spring, the ozone distribution in the Antarctic region is asymmetrical with a maximum in the Australian longitudinal sector and a minimum in the Atlantic longitudes (Grytsai et al., 2005; Agosta and Canziani, 2011). Previous studies have revealed the tendency of the Antarctic polar vortex to exhibit an eastward shift in orientation (Huth and Canziani, 2003), in the ozone minimum location (Grytsai et al., 2005; Malanca et al., 2005; Grytsai et al., 2007a, b; Agosta and Canziani, 2010, 2011; Grytsai, 2011; Hassler et al., 2011) and in the phase of wave 1 in stratospheric temperature (Lin et al., 2010). This eastward shift has been described as possibly connected with a change in tropospheric stationary waves (Grytsai et al., 2007a), tropospheric jet structure (Hio and Hirota, 2002; Agosta and Canziani, 2011) and its strengthening (Wang et al., 2013), and stratospheric ozone and volcanic aerosol concentration (Lin et al., 2010). The quasi-stationary wave (QSW) activity increases typically in austral spring (Randel, 1988), and its enhancement leads to larger vortex asymmetry, a decrease in ozone hole area and net stratospheric ozone loss. It has been noted that the decreased (increased) asymmetry in the ozone distribution is associated with the eastward (westward) phase shift of the zonal minimum from both observations at the southern high latitudes (Grytsai et al., 2008; Agosta and Canziani, 2011) and climate model simulations for the northern high latitudes (Gabriel et al., 2007).

It has been revealed that the Antarctic ozone depletion in spring influences the trend in the Southern Annular Mode (SAM) in summer towards the high-index polarity that leads to a range of significant summertime surface climate changes (Thompson et al., 2011). Recent studies have indicated that a stabilization of the spring ozone depletion has occurred from the mid- or late 1990s (Grytsai, 2011; Salby et al., 2011; Kuttippurath et al., 2013; Dameris and Godin-Beekmann, 2014; Solomon et al., 2016). This stabilization relates to the total area of the ozone hole, mini- mum total ozone values, ozone mass deficit and duration of the ozone hole season. Chemistry-climate models have displayed a general minimum in Antarctic ozone during 20002005 (Siddaway et al., 2013) and slow ozone recovery in the 21st century (Dameris and Godin-Beekmann, 2014). In turn, ozone recovery is expected to continue to impact the SH surface climate (Thompson et al., 2011). This work is focused on the recent tendencies in the zonal asymmetry of the Antarctic total ozone in austral spring and their possible relations to the $\mathrm{SH}$ atmospheric anomalies.

\section{Data and methods}

In this study, gridded monthly mean satellite measurements of the total ozone column (TOC) are used to estimate tendencies in the Antarctic quasi-stationary pattern. We restrict our analysis to the September-November (SON) period (austral spring), when the zonal asymmetry in total column ozone is most pronounced. We use measurements from the Total Ozone Mapping Spectrometer (TOMS)/Nimbus-7 (19791992), TOMS/Earth Probe (1996-2005) and Ozone Monitoring Instrument (OMI) aboard the Aura platform (20062015), which were obtained from the NASA Ozone \& Air Quality website http://www.ozoneaq.gsfc.nasa.gov/. A gap between Nimbus-7 and Earth Probe observations in 19931995 was filled in by Multi-Sensor Reanalysis (MSR) data (http://www.temis.nl; van der A et al., 2010).

The original data are available with $1 \times 1.25^{\circ}$ (TOMS) and $1 \times 1^{\circ}(\mathrm{OMI})$ latitude $\times$ longitude resolution. We averaged the data over each SON interval to suppress the effects of long-period traveling planetary waves (Grytsai et al., 2007a) and selected zonal values from 50 to $80^{\circ} \mathrm{S}$ at $5^{\circ}$ latitude intervals; the centers of our selected latitude bins are 50.5, 55.5, $\ldots 80.5^{\circ} \mathrm{S}$. The zonal asymmetry in the TOC distribution over this latitude range is illustrated by the OMI measurements in October 2014 (Fig. 1a). The thick white contour in Fig. 1a shows the ozone hole boundary, which corresponds to the threshold TOC value of 220 DU (white line on color scale in Fig. 1a) defined by the World Meteorological Organization (WMO) criterion (WMO, 2014); see also (Newman et al., 2004) for the rationale for choosing the $220 \mathrm{DU}$ value.

Figure 1b shows long-term changes in the zonal TOC asymmetry at $65^{\circ} \mathrm{S}$ (white circle in Fig. 1a) during SON 1979-2014. In the 2000s (blue curves in Fig. 1b), when ozone loss culminated, zonal TOC minimum is about 100 DU lower than in the pre-ozone-hole years (red curves) and is notably displaced to the east. This illustrates a general tendency for the zonal TOC minimum to be located further eastward with decreasing minimum level, as noted previously (Grytsai et al., 2005; Lin et al., 2010; Agosta and Canziani, 2011; Hassler et al., 2011). For the quantitative analysis presented here, the longitudes of the zonal TOC maximum and minimum were determined using a $50^{\circ}$ window in longitude to separate out the large-scale parts of 

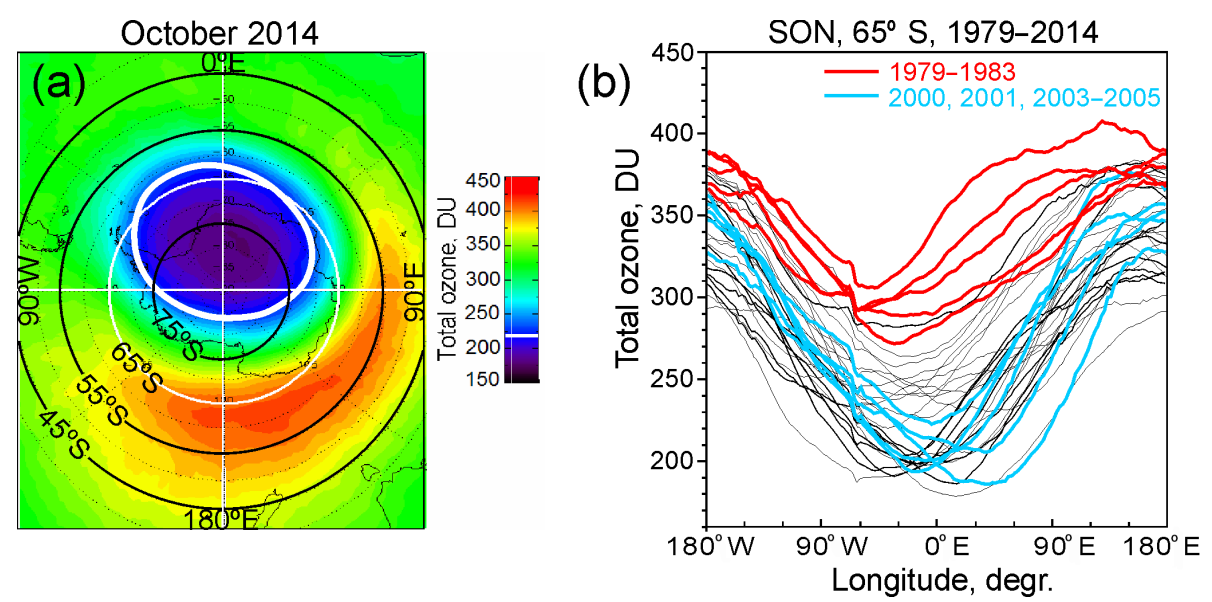

Figure 1. (a) Total ozone at the SH middle/high latitudes in October 2014. (b) Zonal dependence of the total ozone at $65^{\circ} \mathrm{S}$ averaged for September-November for each year between 1979 and 2014; red curves show pre-ozone-hole years 1979-1983, and blue curves show the years of maximum ozone hole: 2000-2005 (apart from the anomalous 2002). Modified from Grytsai et al. (2007a).

the disturbances. In this work, long-term tendencies were obtained from polynomial approximation calculated with a least-squares method. The calculation method is described in Supplement 1.

Regression, correlation and composite analyses were used to relate the QSW TOC minimum $\left(\mathrm{QSW}_{\min }\right)$ longitude to the meteorological variables. The reliability of the main results is examined comparing the relationships with the two reanalysis data. We use gridded atmospheric variables from the European Centre for Medium-Range Weather Forecasts (ECMWF) ERA-Interim reanalysis (Dee et al., 2011; http://www.ecmwf.int/en/research/climate-reanalysis/ era-interim) at $1.5^{\circ} \times 1.5^{\circ}$ (longitude $\times$ latitude) resolution and NCEP-NCAR reanalysis (Kalnay et al., 1996; http:// www.esrl.noaa.gov/psd/) at $2.5^{\circ} \times 2.5^{\circ}$ resolution.

\section{Results}

\subsection{Longitudinal changes in the QSW structure}

First, we compare the long-term changes in total ozone (Fig. 2a) and the longitudinal position of the quasi-stationary ozone minimum (Fig. 2b) at $65^{\circ} \mathrm{S}$ latitude, which is located in the edge region of the ozone hole and polar stratospheric vortex (Roscoe et al., 2012) and where the largest QSW amplitude is observed (Grytsai et al., 2007a; Ialongo et al., 2012).

Cubic polynomial fits are shown by thick curves in Fig. 2. These fits are included to highlight the long-term variations in each of the time series and are not done in consideration of any particular underlying physical process. In the early 2000s, the decadal tendencies indicated by the slope of the polynomial fit changed sign both in ozone (from decreasing levels to increasing levels, Fig. 2a) and in ozone minimum longitudes (from eastward shift to westward shift, Fig. 2b).
Comparison of polynomials from $k=2$ to $k=6$ gives similar opposite tendencies before and after the early 2000s (see Supplement 2 and Fig. S1). Note that the quasi-stationary maximum in total ozone at $65^{\circ} \mathrm{S}$ shows a relatively small decadal shift in longitude (Fig. 2c, thick curve) compared with the level of interannual variability and changes in the $\mathrm{QSW}_{\text {min }}$ longitude.

On comparing Fig. 2a and b, there appears to be some consistency in the epoch of inflexion in the tendencies of both the column amount and the maximum eastward longitude shift of the zonal TOC minimum (around 2000). The eastward shift in the QSW structure over Antarctica has been described previously (Huth and Canziani, 2003; Grytsai et al., 2005, 2007a; Malanca et al., 2005; Agosta and Canziani, 2010, 2011; Lin et al., 2010; Grytsai, 2011; Hassler et al., 2011). Eastward shift speeds of about 15$20^{\circ}$ decade $^{-1}$ are consistent among various studies (Grytsai et al., 2007a; Lin et al., 2010; Hassler et al., 2011). For the period 1979-2000, the time series in Fig. 2b gives a linear trend of $14.4 \pm 12.5^{\circ}$ decade $^{-1}$, significant at the $95 \%$ level. The westward shift between the early 2000 s and early 2010 s apparent in Fig. 2b is statistically insignificant, and a longer time series is necessary to reliably establish this tendency. Note that Fig. $2 \mathrm{~b}$ shows large longitude variations during some of the most recent years. Interannual changes in the 2000s and early 2010s covered a wider longitudinal range than in the previous decades. For example, the position of the quasi-stationary minimum was near its extreme western values in 2011 and 2013, whereas it reached the farthest eastern longitude in 2010 .

Long-term tendencies in the QSW minimum/maximum longitudes at the seven latitudes between 50 and $80^{\circ} \mathrm{S}$ are illustrated in Fig. 3. The extreme longitudinal departures for 1979, 2002 and 2015 relative to the cubic polynomial are shown by closed circles in Fig. 2b. A significant eastward 
(a)

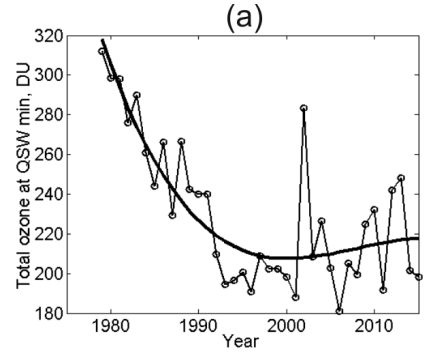

(b)

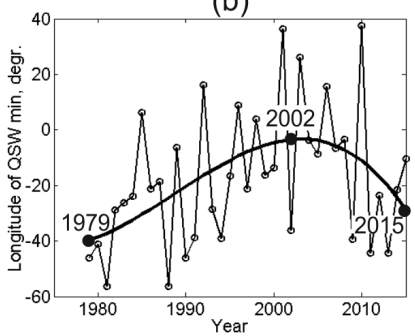

(c)

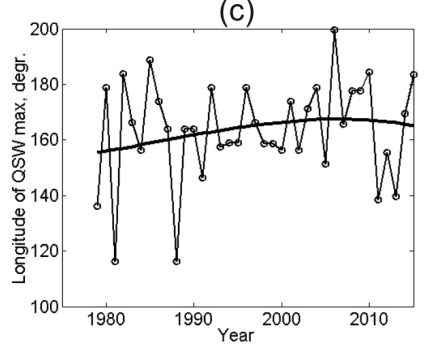

Figure 2. (a) Total ozone at zonal QSW minimum, (b) longitude of QSW minimum and (c) longitude of the QSW maximum at $65^{\circ} \mathrm{S}$ averaged for September-November. Thin lines are time series of 1979-2015, and thick lines are the third-degree polynomial fits. Longitudes for 1979, 2002 and 2015 from polynomial fitting are marked in (b) by closed circles.

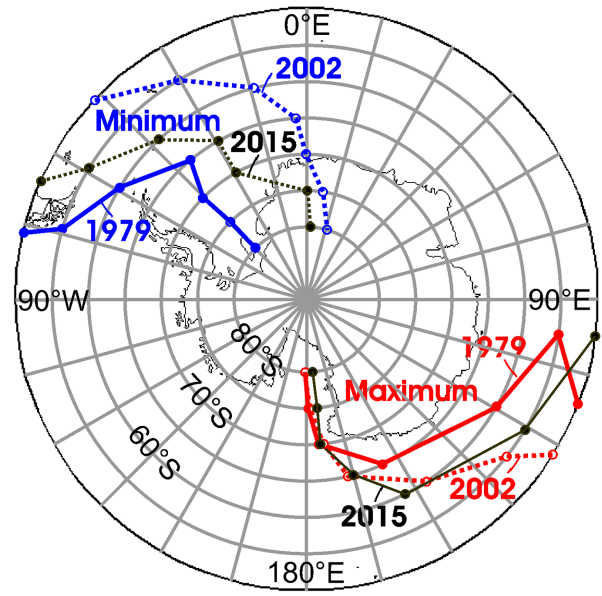

Figure 3. Map of longitudinal locations of zonal QSW maximum (red) and zonal QSW minimum (blue) at seven latitudes between 50 and $80^{\circ} \mathrm{S}$; westernmost (easternmost) longitudes in 1979 (2002) determined from the polynomial fit, as shown in Fig. $2 b$, relate to solid (dotted) lines. Black lines mark longitudes for 2015. Modified from Grytsai et al. (2007a).

shift of the QSW min $_{\text {from }} 1979$ (solid blue curve) to the early 2000s (dotted blue curve for 2002) by 30-60 degrees of longitude is seen. The curve for 2015 in the region of the zonal TOC minimum (black dotted curve in Fig. 3) is located between the curves for 1979 and 2002 and is shifted westward over the whole zone $\left(50-80^{\circ} \mathrm{S}\right)$, i.e., in the opposite direction compared with most years of the preceding decade. Changes of the QSW maximum longitudinal position are not regular (Grytsai et al., 2007a), and the largest eastward shifts are seen only at 55 and $60^{\circ} \mathrm{S}$; however, they are not statistically significant due to strong interannual variability (Fig. 2c). The relative stability of the zonal maximum location suggests that higher zonal wave numbers, quasi-stationary wave 2 (QSW2) and wave 3 (QSW3), could be present in the QSW structure, in addition to the dominant quasi-stationary wave 1 (QSW1) (Grytsai et al., 2007a; Agosta and Canziani, 2011).

The two curves in Fig. 4 illustrate the similarity in the interannual variations and decadal changes of the $\mathrm{QSW}_{\min }$

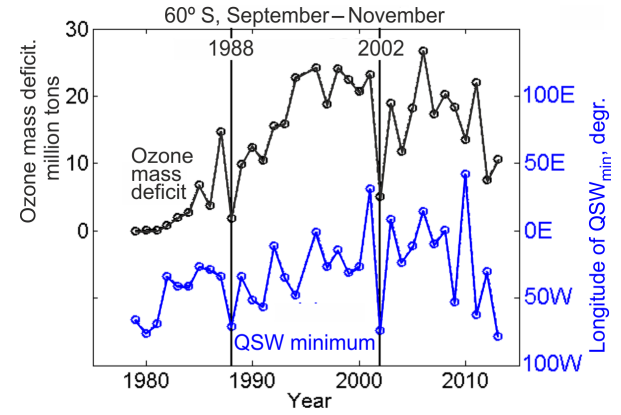

Figure 4. Ozone mass deficit and position of the quasi-stationary minimum in the ozone distribution at $60^{\circ} \mathrm{S}$ averaged over September-November. Pearson's correlation coefficient between the two time series is $r=0.57$. Data for the ozone mass deficit at the $\mathrm{SH}$ high latitudes are from http://ozonewatch.gsfc.nasa.gov/ meteorology/SH.html. Years of large (1988) and extreme (2002) stratospheric warmings are indicated by vertical lines.

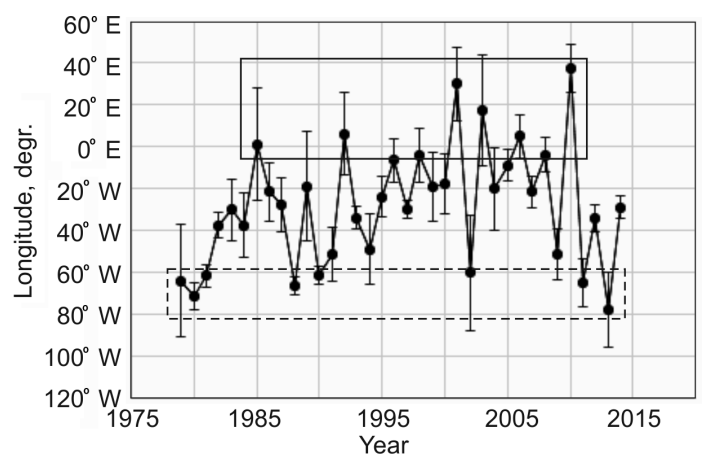

Figure 5. Longitude of MSR TOC ozone minimum $\left(\mathrm{QSW}_{\min }\right)$ for SON averaged over four latitudes between 55 and $70^{\circ} \mathrm{S}$, inclusive. The vertical bars span \pm 1 standard deviation of the individual values used in each average. The solid (dashed) rectangle outlines the upper 80th percentile (lower 20th percentile), corresponding to the maximal eastward (westward) shift of the $\mathrm{QSW}_{\text {min }}$ longitude.

longitudes and ozone mass deficit in Antarctic spring. The linear correlation between the two variables is positive and $r=0.49-0.57$ for the seven latitude circles between 50 and 
$80^{\circ} \mathrm{S}$ with maximum at $60^{\circ} \mathrm{S}$. The correlation coefficient $r$ was calculated for the time series length $N=35$ (1979-2013, Fig. 4), and the value $r=0.43$ is significant at the $99 \%$ confidence limit based on a two-tailed Student's $t$ test. Hence, an eastward shift of the QSW minimum in the ozone distribution with high probability corresponds to a greater ozone mass deficit (larger ozone loss).

Simultaneous negative deviations are observed in the years of large (1988) and major (2002) stratospheric warmings (vertical lines in Fig. 4). Both anomalous events in the SH stratosphere were associated with enhanced planetary wave activity (Varotsos, 2003a; Allen et al., 2003; Baldwin et al., 2003; Grytsai et al., 2008). As seen from Fig. 4, relatively small ozone mass deficits (high total ozone levels) correspond to the westward shift of the $\mathrm{QSW}_{\min }$ position. Note that this correspondence is not observed in some recent years; the Antarctic spring in 2010 is characterized by eastern longitude of the $\mathrm{QSW}_{\min }$ at low ozone mass deficit, and the relationship for 2011 is opposite.

The results of Figs. 2-4 show that the eastward shift in the TOC zonal minimum longitude in the Antarctic region occurred during the 1980s-1990s and was accompanied by rapid and intense ozone loss. This decadal tendency appears to have stopped in the early 2000s and became of possibly reverse sign later in the 2000s and 2010s. Generally, the behavior of the zonal TOC minimum in Figs. $2 b$ and 3 follows the decadal change in the severity of the ozone hole due to international controls on ozone-depleting substances (ODSs; Salby et al., 2011; Solomon et al., 2016), with increasing depletion of the Antarctic ozone in the 1980s and 1990s, and its leveling off and the possible start of recovery in the 2000s2010s (Siddaway et al., 2013). Significant decadal changes in the SH polar ozone are coupled with the stratospheric thermal regime (e.g., Crook et al., 2008); because of the zonal asymmetry in the ozone heating, they impact planetary wave propagation (Albers and Nathan, 2012) and regional climate change in both the troposphere and the stratosphere (Gillett et al., 2009; Waugh et al., 2009). Couplings between changes in the QSW structure in Antarctic total column ozone and in atmospheric variables are analyzed below.

\subsection{Relationships between the QSW minimum longitude and meteorological parameters}

To determine the most reliable mean tendencies, we have created a time series for the $\mathrm{QSW}_{\text {min }}$ longitudes averaged between 55 and $70^{\circ} \mathrm{S}$ (four latitude circles in Fig. 3); this is shown in Fig. 5. Bars in Fig. 5 indicate standard deviations for each year, and they are generally relatively small compared with the level of interannual variability in the time series.

We next consider the regression between the time series of Fig. 5 and SON average climatological anomalies of ERAInterim meteorological variables. We first produce monthly climatological anomalies for each gridded monthly average variable at the native horizontal resolution by subtracting the associated long-term monthly mean (over 1979-2014 for ERA-Interim and 1981-2010 for NCEP-NCAR). We then produce averages of the anomalies in grid boxes of $10^{\circ} \times 10^{\circ}$ (latitude $\times$ longitude) over the SON months of each year. Finally, we evaluated the regression coefficient (RC) between

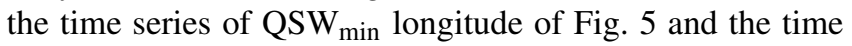
series of SON climatological anomalies at each location. Figure $6 \mathrm{a}$ and $\mathrm{b}$ present the global distributions of the regressions for surface pressure (SP) and $2 \mathrm{~m}$ air temperature ( $T-2 \mathrm{~m})$ over 1979-2014, respectively. Dots with numbers from 1 to 5 in Fig. 6a are placed at each grid box center where the linear correlation coefficient is significant at the $95 \%$ confidence limit based on Student's $t$ test (boxes with significant correlations are also diagonally hatched) and where the fraction of variance explained (values in $\%$ ) is $\geq 25 \%$ (see text below).

The RC distributions as in Fig. 6a and b but for RC between the $\mathrm{QSW}_{\text {min }}$ longitude and ERA-Interim $200 \mathrm{hPa}$ climatological anomalies of zonal wind speed (U200) and vertical pressure wind speed (W200) are shown in Fig. 6c and $\mathrm{d}$, respectively. The pressure level of $200 \mathrm{hPa}$ corresponds to the upper troposphere in the tropics and lower stratosphere in the SH extratropics and is usually used to analyze the interaction between the tropics and extratropics (e.g., Mo and Higgins, 1998). The RC distribution for sea surface temperature (SST) is similar to that for $T-2 \mathrm{~m}$ (Fig. 6b), and the $\mathrm{RC}$ distribution for meridional wind V200 is similar to that for $W 200$ (Fig. 6d), and are not shown here.

The RC distribution in Fig. 6a shows an annular pattern that is similar to a classic Southern Annular Mode pattern in SH climate variability, with pressure or geopotential height anomalies of opposite sign at the middle and high latitudes (Thompson and Wallace, 2000). Negative (positive) regression coefficients at the high (middle) SH latitudes indicate that the QSW $_{\text {min }}$ eastward shift is associated with decreased (increased) surface pressure, i.e., with the SAM deviation towards positive polarity.

A positive polarity of the annular mode is accompanied by strengthening of the subpolar westerlies in the SH troposphere and stratosphere and cooling of polar cap regions (Thompson and Wallace, 2000). The RC maximum around $60^{\circ} \mathrm{S}$ in Fig. $6 \mathrm{c}$ and the RC minimum over the Antarctic continent (poleward of $60^{\circ} \mathrm{S}$ ) in Fig. $6 \mathrm{~b}$ display similar tendencies in the relationships between the $\mathrm{QSW}_{\text {min }}$ eastward shift and increase of $U 200$ and decrease of $T-2 \mathrm{~m}$, respectively.

Zonally asymmetric components of the SH circulation, which are most marked in the austral winter and spring (Mo and Higgins, 1998; Fogt et al., 2012a), are also presented in Fig. 6. Three positive RC anomalies at the SH midlatitudes (grid boxes 1, 2 and 3 in Fig. 6a) demonstrate the presence of a QSW3 structure. The highest negative RC anomaly between grid boxes 2 and 3 is spatially close to the subpolar negative anomaly at grid box 4 and is possibly combined effect of QSW1 and QSW3 (Mo and Higgins, 1998). A signif- 

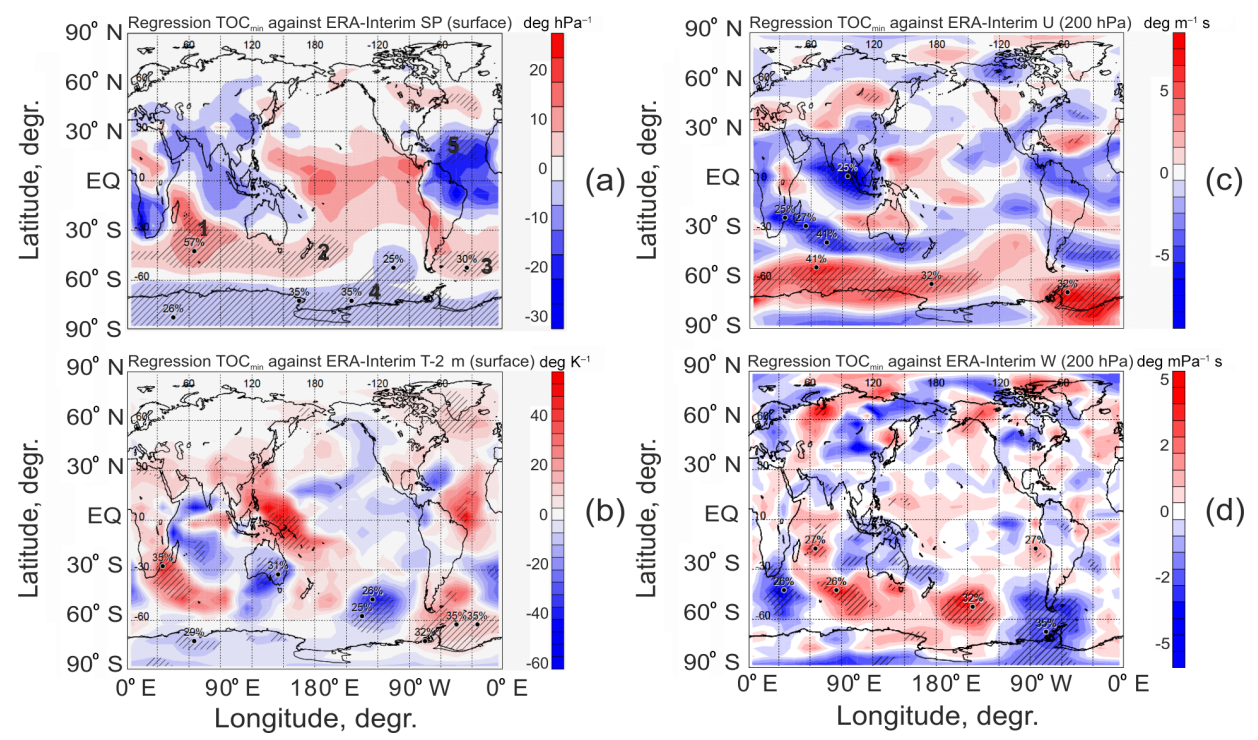

Figure 6. Regression coefficient of the TOC QSW minimum longitude against ERA-Interim climatological anomalies of (a) surface pressure (SP), (b) $2 \mathrm{~m}$ air temperature $(T-2 \mathrm{~m}$ ), (c) $200 \mathrm{hPa}$ zonal wind speed (U200) and (d) $200 \mathrm{hPa}$ vertical pressure wind speed (W200) for SON 1979-2014. The units are degrees of longitude per (a) $\mathrm{hPa}$, (b) $\mathrm{K}$, (c) $\mathrm{m} \mathrm{s}^{-1}$ and (d) $\mathrm{mPa} \mathrm{s}^{-1}$. The hatching is shown where the correlation between the two variables in each panel is significant at the $95 \%$ confidence limit.

icant negative RC anomaly near West Antarctica (grid box 4 in Fig. 6a with an explained variance of $35 \%$ ) is spatially coincident with the "pole of variability" in the AmundsenBellingshausen Sea low (ABSL) region (Fogt et al., 2012b, and references therein; Turner et al., 2013; Raphael et al., 2016). The midlatitude QSW3 patterns extended to subAntarctic latitudes are seen also in the RC distribution for $T-2 \mathrm{~m}$ and $W 200$ (Fig. 6b and d, respectively).

The presence of the QSW3 structure in Fig. 6b introduces regional anomalies into the surface temperature distribution. The patterns suggest that, when the $\mathrm{QSW}_{\text {min }}$ moves to the east, surface temperatures are warmer in the Antarctic Peninsula-Weddell Sea region and in the southwest area of Indian Ocean, and cold anomalies appear in the South Pacific and southeast Australia. Hence, variability in zonal asymmetry in the Antarctic ozone during the spring months, with high probability, is indicative of the $\mathrm{SH}$ regional climate variability.

Zonal asymmetry in the SH troposphere circulation is closely coupled with the Pacific-South American (PSA) mode (Mo and Higgins, 1998). The PSA pattern in the RC distribution in Fig. 6 is of insignificant intensity, whereas pronounced meridional wave trains are seen in the IndianAustralian sector and Atlantic-South American sector (U200 in Fig. 6c). As follows from the relationships below, combined wave activity over the three ocean basins can contribute to the $\mathrm{QSW}_{\min }$ longitude variability.

All patterns of Fig. 6 are reproduced in correlations with the same variables using the NCEP-NCAR reanalysis data (Supplement 3, Fig. S2), confirming the reliability of the results. In general, Fig. 6 and Supplement Fig. S2 show that interannual variations of the SON QSW min $_{\text {longitude during }}$ 1979-2014 are associated with the zonally symmetric annular mode and zonally asymmetric QSW structures in the SH atmosphere. In Fig. 7 we present anomaly composites (averages) for years of extreme western (lower 20th percentile) and eastern (upper 20th percentile) $\mathrm{QSW}_{\text {min }}$ longitudes to further investigate the patterns shown in Fig. 6. Monthly mean anomalies for September, October and November were calculated by subtraction of the climatological means of 1979-2014 from the monthly mean variable value in each grid box as described above concerning Fig. 6. Then monthly mean anomalies were averaged over the SON months.

Anomaly composites for the lower 20th percentile of the $\mathrm{QSW}_{\min }$ longitudes $\left(<-60^{\circ}\right.$ longitude, eight westernmost locations outlined by dashed rectangle in Fig. 5) are presented in Fig. 7a-c. Anomaly composites for the higher 20 th percentile $\left(>-3.8^{\circ}\right.$ longitude, eight easternmost locations outlined by solid rectangle in Fig. 5) are presented in Fig. 7d-f. The years for the westernmost longitudes are 1979, 1980, 1981, 1988, 1990, 2002, 2011 and 2013 (left column of Fig. 7), and the years for the easternmost longitudes are 1985, 1992, 1998, 2001, 2003, 2006, 2008 and 2010 (right column of Fig. 7).

It generally is seen from Fig. 7 that transition from the westernmost longitudes (left column) to the easternmost longitudes (right column) is accompanied by the reversal in the sign of the anomalies. The western (eastern) longitudes correspond to negative (positive) zonal wind anomaly around $60^{\circ} \mathrm{S}$ in Fig. 7a (Fig. 7d) and positive (negative) surface pressure poleward of $60^{\circ} \mathrm{S}$ in Fig. 7b (Fig. 7e). Opposite anomaly combinations appear at the SH middle latitudes. 
(a)

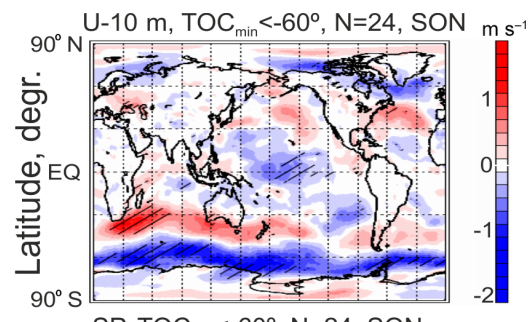

(b)

(c)
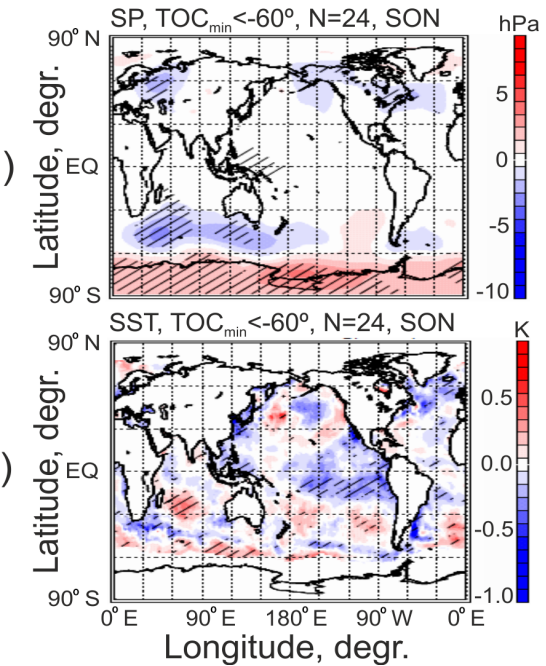

(d)

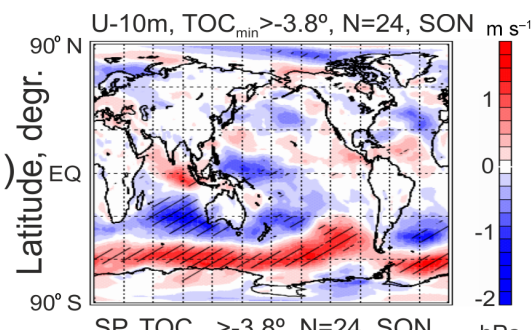

(e)

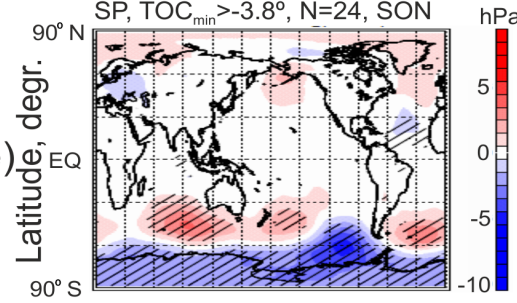

(f)

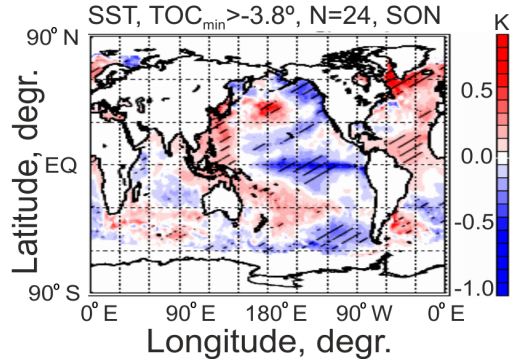

Figure 7. Anomaly composites with respect to the mean climatology for 1979-2014 of ERA-Interim surface meteorological variables for (left) the lower 20th percentile of mean SON QSW $\min$ longitudes (western phases) and (right) the upper 20th percentile of mean SON

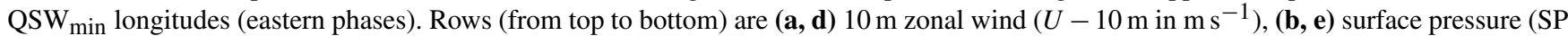
in $\mathrm{hPa}$ ) and (c, f) sea surface temperature (SST in K). Diagonal shading indicates regions significant at the $95 \%$ confidence limit (evaluated by comparing the value of each grid box with its standard deviation).

These changes are consistent with the regression maps in Fig. 6a and c, where eastward phase shift indicates similar relationships with $U 200$ (Fig. 6c) and SP (Fig. 6a) displaying the SAM pattern.

Note that the SP anomaly composites show the QSW3like structure at the $\mathrm{SH}$ midlatitudes, which is more intense and shifted to the east in the case of the easternmost $\mathrm{QSW}_{\text {min }}$ longitudes than in the case of the westernmost longitudes (Fig. 7e and b, respectively). The pattern of Fig. 7e means that the regional surface pressure becomes higher in the eastward-shifted elements of the QSW3 structure; i.e., it deviates to anticyclonic regime in the case of the easternmost $\mathrm{QSW}_{\text {min }}$ longitudes. The surface pressure growth between the QSW3 anomalies in Fig. 7b and e equates to about $8 \mathrm{hPa}$. Given the seasonal timescale (September-November), this can contribute significantly to regional surface climate change in spring associated with the ozone hole asymmetry change.

The SST anomaly composites demonstrate a relationship of the extreme $\mathrm{QSW}_{\text {min }}$ longitudes with different tropical regions: western (eastern) longitudes in Fig. 7c (Fig. 7f) are observed for the negative SST anomalies in the eastern (central) tropical Pacific. The easternmost longitudes in Fig. $7 \mathrm{f}$ demonstrate the positive SST anomalies in the western tropi- cal Pacific and in the Atlantic, similarly to the same anomaly locations in $T-2 \mathrm{~m}$ in Fig. 6b. Such positive anomaly distributions resemble the pattern of the anomalous SST trend calculated with zonal mean trend removed (Schneider et al., 2015, their Fig. 2f) and the pattern of Atlantic Multidecadal Oscillation (Li et al., 2014; their Fig. 1e).

The SAM, QSW3 and SST patterns seen from Fig. 7 are fully reproduced with the same variables of the NCEPNCAR reanalysis (Supplement 3, Fig. S3). This is evidence that the $\mathrm{QSW}_{\min }$ longitudes in the total ozone are steadily coupled with the climate mode (SAM) and stationary wave structure (QSW3) in the SH atmosphere, as well as with the specific SST anomaly pattern. Further evidence of this coupling is seen from Fig. 8.

Regression of the $\mathrm{QSW}_{\min }$ longitudes against the $\mathrm{SP}$ anomalies at grid box $1\left(42.5^{\circ} \mathrm{S}, 65.0^{\circ} \mathrm{E}\right.$; Fig. 6a), a midlatitude element of QSW3, is shown in Fig. 8a. A close similarity can be seen in variations of the SP anomalies in grid box 1 and the $\mathrm{QSW}_{\min }$ longitudes averaged over $55-70^{\circ} \mathrm{S}$ : the square of the linear correlation coefficient is $r^{2}=0.57$. In Fig. 8b, time series of the $\mathrm{QSW}_{\text {min }}$ longitude and the standardized SAM index (Marshall, 2003; https://climatedataguide.ucar.edu/climate-data/ marshall-southern-annular-mode-sam-index-station-based) 

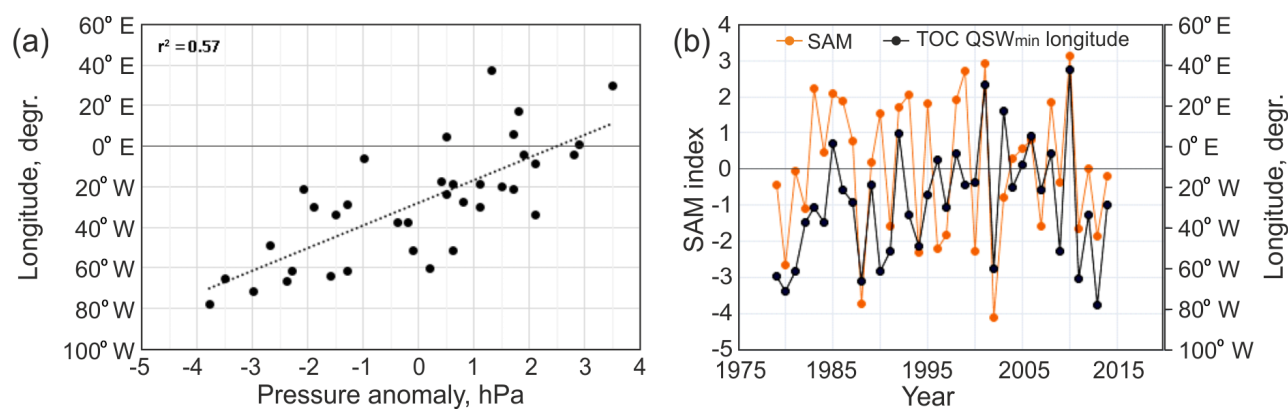

Figure 8. (a) Regression of surface pressure anomaly for grid box $1\left(42.5^{\circ} \mathrm{S}, 65.0^{\circ} \mathrm{E}\right)$ of Fig. 6a (horizontal axis) against the QSW $\mathrm{min}$ longitude (vertical axis). (b) Time series of the standardized SAM index (orange, left axis) and the QSW min longitude (black, right axis). The time series of the $\mathrm{QSW}_{\min }$ longitude is that shown in Fig. 5. All variables are averaged over September-November. The $r^{2}$ value is significant at the $95 \%$ confidence limit.

are shown. The square of the linear correlation coefficient between the two time series $r^{2}=0.35$. Therefore, approximately 57 and $35 \%$ of the $\mathrm{QSW}_{\text {min }}$ longitude variance (both significant at the $95 \%$ confidence limit) can be explained by the surface pressure anomaly variance described by regional (grid box 1, QSW3 pattern) and hemisphericscale (SAM) indices, respectively. This indicates that a significant interaction takes place between the identified anomalies in the tropospheric circulation and zonal asymmetry in total ozone (see Sect. 4 for discussion on the SH troposphere-stratosphere interaction).

As noted from Fig. 6c, the regression coefficient distribution for $U 200$ shows meridional wave trains in the Indian and Atlantic sectors. To contrast the result of Fig. 6c, anomaly composites as in Fig. 7 but for $200 \mathrm{hPa}$ are presented in Fig. 9. Similarly to Fig. 7, transition from the westernmost QSW $_{\min }$ longitudes to easternmost ones (left and right columns, respectively) is accompanied by the anomaly sign reversal. The $U 200$ anomaly composites in Fig. 9b and f show meridional wave train patterns over the three ocean basins, albeit without clear eastward propagation, and it is difficult to determine the wave train sources. Relation of the $\mathrm{QSW}_{\text {min }}$ longitude to these wave patterns means combined contribution of the QSW sources to the wave structure in the SH stratosphere.

It is important to note the eastward shift of the anomalies between the westernmost and easternmost $\mathrm{QSW}_{\text {min }}$ longitudes. It can be seen comparing, for example, the QSW1 patterns in $T 200$ at the middle-high SH latitudes (Fig. 9a and e) and the QSW3 patterns in W200 at the middle-subAntarctic latitudes (Fig. 9c and g). Note also more intense anomalies for the easternmost $\mathrm{QSW}_{\text {min }}$ longitudes in Fig. 9 (right column), which demonstrate lower temperature over large part of the middle-high SH latitudes (T200, Fig. 9e), stronger sub-Antarctic zonal circulation in the lower stratosphere (U200, Fig. 9f), and enhancement of the QSW3 patterns in vertical velocities (W200, Fig. 9g) and eddy heat flux $\left(\mathrm{V}^{\prime} \mathrm{T}^{\prime} 200\right.$, Fig. 9h). Since the easternmost longitudes charac- terize mainly the 1990s and 2000s (as outlined by solid rectangle in Fig. 5), the right column in Fig. 9 could indicate that the 2 decades of the strongest ozone loss were favorable for the closest interaction between the SAM/QSW3-related anomalies in the SH atmosphere and the QSW structure in total ozone. As in the case of Fig. 7, the NCEP-NCAR reanalysis shows very similar patterns in the same anomaly composites for $200 \mathrm{hPa}$ (Supplement 3, Fig. S4), confirming the reliability of the results of Fig. 9 .

In connection between the changes in the QSW structure in total ozone and atmospheric parameters, the clear eastward shift in the QSW3 patterns at the SH midlatitudes (Fig. 7b and $\mathrm{e}$ and Fig. 9c and g) is of particular interest. This tendency is analyzed in more detail using the correlative relationships between the $\mathrm{QSW}_{\min }$ longitude and air temperature.

\subsection{Correlations between the $\mathrm{QSW}_{\text {min }}$ longitude and the air temperature}

The linear correlation between time series of the $\mathrm{QSW}_{\text {min }}$ longitude at $65^{\circ} \mathrm{S}$ and the ERA-Interim air temperature was evaluated. As the QSW3 structure is concentrated at the midand sub-Antarctic latitudes (Figs. 6, 7 and 9), the correlation for air temperature averaged over the zone $40-60^{\circ} \mathrm{S}$ was calculated. In Fig. 10, the results in the longitude-height cross section for the 36-year period 1979-2014 are presented. The sample sizes in this case mean that correlation $r=0.33$ is significant at the $95 \%$ confidence limit (black and white contours in Fig. 10 for the positive and negative correlations, respectively).

A clear separation between the QSW1 pattern above the tropopause (peak values of $r \approx \pm 0.7$; climatological thermal tropopause shown by black curve) and the QSW3 pattern below the tropopause $\left(r_{\max } \approx 0.5\right)$ is seen.

The strong correlation in the stratosphere (between 200 and $20 \mathrm{hPa}$, or 12 and $26 \mathrm{~km}$, respectively, in Fig. 10), firstly, demonstrates close coupling between the $\mathrm{QSW}_{\min }$ longitude and the stratospheric temperature in their interannual vari- 
(a)

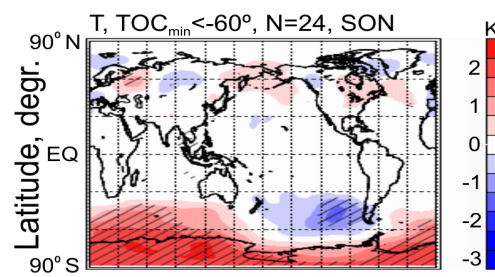

(b)

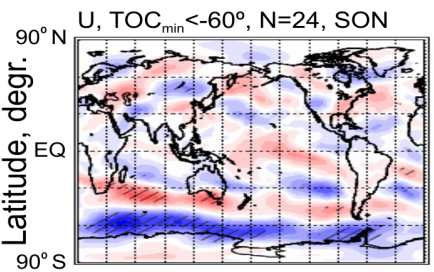

(c)
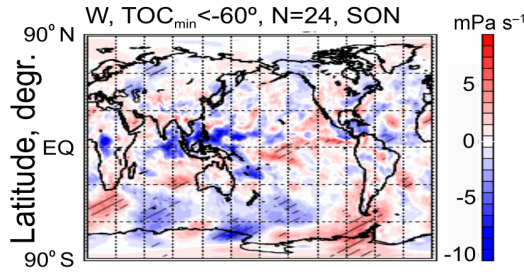

$\mathrm{VT}, \mathrm{TOC}_{\min }<-60^{\circ}, \mathrm{N}=24, \mathrm{SON}$

(d)

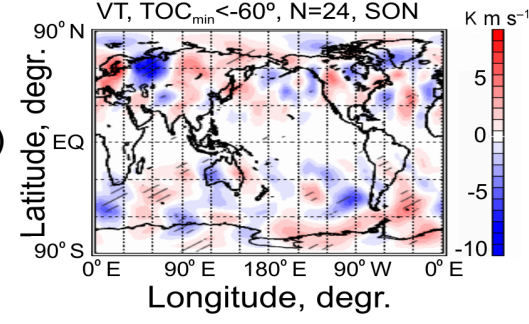

(e)

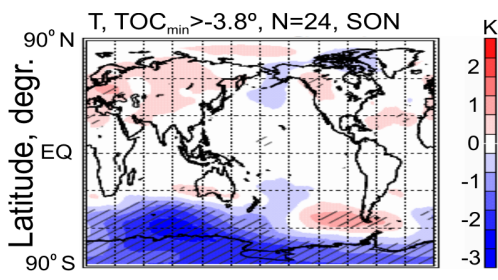

(f)
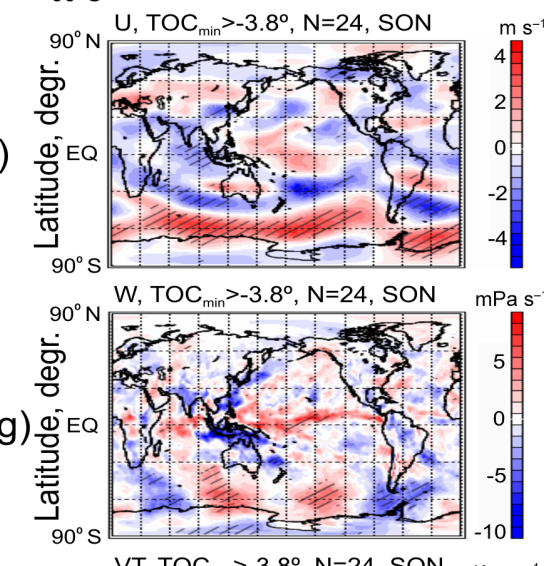

(h)

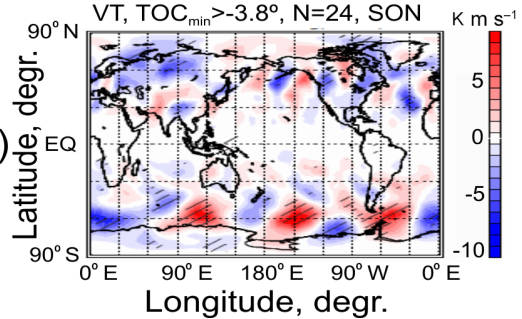

Figure 9. As in Fig. 7 but for ERA-Interim meteorological variables at $200 \mathrm{hPa}$ (a, e) air temperature ( $T 200$ in K), (b, f) zonal wind (U200 in $\left.\mathrm{m} \mathrm{s}^{-1}\right),(\mathbf{c}, \mathbf{g})$ vertical pressure wind $\left(W 200\right.$ in $\left.\mathrm{mPa} \mathrm{s}^{-1}\right)$ and $(\mathbf{d}, \mathbf{h})$ eddy heat flux $\left(V^{\prime} T^{\prime}\right.$ in $\left.\mathrm{K} \mathrm{m} \mathrm{s}^{-1}\right)$. Diagonal shading indicates the same as in Fig. 7.

ations and, secondly, confirms that the $\mathrm{QSW}_{\text {min }}$ longitude variability is mainly due to the QSW1 contribution. As could be seen from Fig. S5 in Supplement 3, the correlation using the NCEP-NCAR temperature is almost identical to the result of Fig. 10 with the ERA-Interim temperature.

An important feature of the QSW3 pattern in the troposphere is its altitudinal location: the three correlation maxima are located predominantly in the middle troposphere, and their peak values are at about $500 \mathrm{hPa}$ (Fig. 10). The correlation is significantly lower at $1000 \mathrm{hPa}$, which could explain relatively weak SST anomalies at the SH midlatitudes in Fig. 7c and f. Therefore, the mid-tropospheric pressure level of $500 \mathrm{hPa}$ was chosen to search for possible decadal changes in the correlations between the anomalies in air temperature and the $\mathrm{QSW}_{\min }$ longitudes. In Fig. 11, correlation coefficient distributions in a sequence of the five 14-year intervals with the 5-6-year steps are shown. The sample size $N=14$ and calculated lag-one autocorrelations give significance level of $95 \%$ for the correlation coefficient $r=0.51$.

It is seen that the significant positive correlation peaks (black contours in Fig. 11) shift eastward between 19791992 and $1990-2003$ by $30-60^{\circ}$ (thick lines in Fig. 11a-c).
Later, they remain on average at the easternmost longitudes in 1995-2008 and 2001-2014 (Fig. 11d and e, respectively). The QSW3 peak near $180^{\circ} \mathrm{E}$ exhibits a shift in opposite direction in the last time interval (Fig. 11e). Correlation sequence with the NCEP-NCAR T500 field displays similar tendencies (Supplement 3, Fig. S6).

A significant negative anomaly of the correlation coefficient $(r<-0.5$, white contours) in the South Pacific extending to polar latitudes into the ABSL region is longitudinally steady (white dashed line). As noted above concerning similar anomaly location in the RC distributions in Fig. 6a and b, this could be a combined effect of QSW1 and QSW3. Here, meteorological variables in the troposphere are correlated with the $\mathrm{QSW}_{\text {min }}$ longitude in total ozone in their interannual variations but do not show consistent decadal changes in spatial pattern. Note that annual cycle in the decadal shift of the ABSL longitude could contribute to distinction in zonal shift of the correlation anomalies at the middle and high SH latitudes in Fig. 11. The ABSL shifted westward on average by $-5^{\circ}$ decade $^{-1}$ in September-November 1979-2008 (Turner et al., 2013, their Fig. 13), which could partly compensate for 

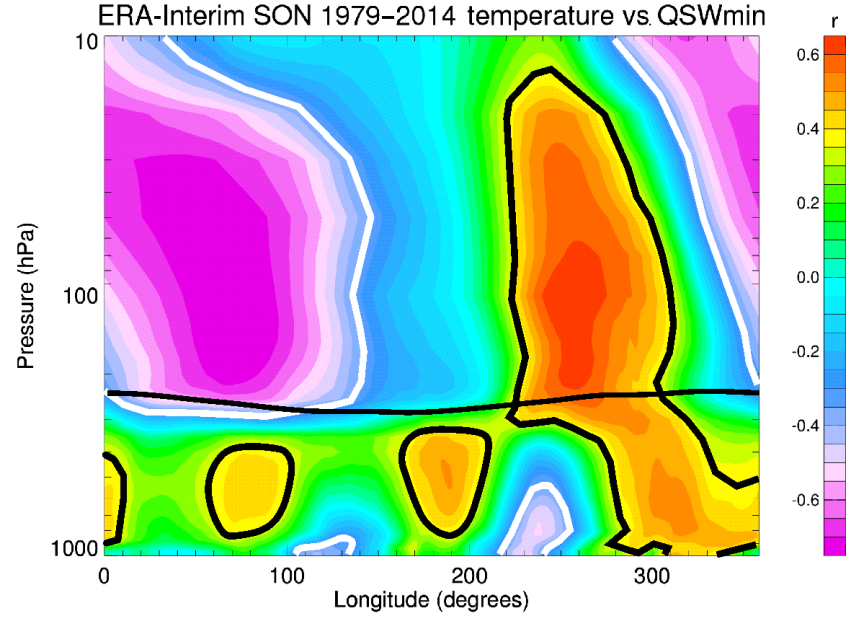

Figure 10. Longitude-height cross section of the correlation between the $\mathrm{QSW}_{\min }$ longitude at $65^{\circ} \mathrm{S}$ and ERA-Interim air temperature averaged over the zone $40-60^{\circ} \mathrm{S}$ for SON 1979-2014. Thick black curve marks climatological thermal tropopause. Black (white) contours show positive (negative) correlations significant at the $95 \%$ confidence limit.

the eastward shift observed in the correlation maxima of the adjoining midlatitudinal zone (Fig. 11).

It can be summarized that common decadal tendencies in total ozone (Fig. 2a), QSW minimum location in the total ozone (Fig. 2b), QSW1 pattern in the lower stratosphere (Fig. 9a and e), and QSW3 pattern in the troposphere (Figs. 7b, e and 11) and lower stratosphere (Fig. 9c and g) exist. On an interannual timescale, the SAM- and QSW1/QSW3-like patterns in the SH circulation associated with variability in the QSW minimum longitude in total ozone are dominating.

In general, the results of Sect. 3 show that the $\mathrm{QSW}_{\min }$ longitude changes in the TOC distribution are in statistically significant associations with both the zonal mean (SAM pattern) and regional (QSW1 and QSW3 patterns) anomalies in the spring $\mathrm{SH}$ atmosphere. These associations allow us to identify the SH regions where the climate changes in spring are accompanied by ozone asymmetry changes. Our results, thus, have two differences from the known impact of the spring ozone loss on the summer SH climate (Thompson et al., 2011). First, quantitative relationships (Figs. 6-11) are not built on the ozone level changes but on a spatial parameter, which describes the changes in the location of the zonally asymmetric ozone anomaly (the $\mathrm{QSW}_{\min }$ longitude). Second, revealed climate signals characterize the Antarctic spring (September-November) and are associated with simultaneous evolution of the ozone hole asymmetry. Possible features of the troposphere-stratosphere interaction, which could contribute to occurrence of revealed links, are discussed below.

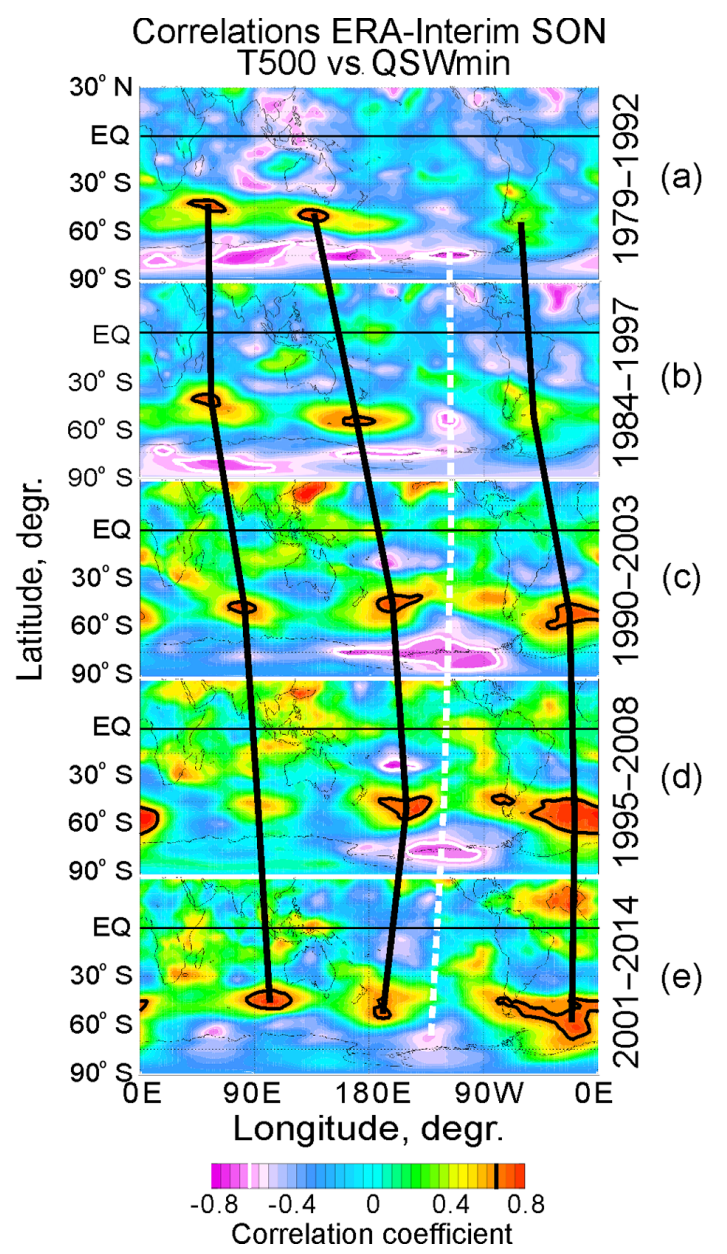

Figure 11. Correlation between the $\mathrm{QSW}_{\text {min }}$ longitude at $65^{\circ} \mathrm{S}$ and ERA-Interim air temperature at $500 \mathrm{hPa}$ south of $30^{\circ} \mathrm{N}$. Five sequential 14-year intervals with 5-6-year steps are presented. Black (white) contours show positive (negative) correlation peaks $r=0.65$ significant at the $99 \%$ confidence limit. Thick solid black (dashed white) lines mark mean longitudinal positions of the positive (negative) correlation peaks in the QSW3 structure.

\section{Discussion}

The evolution of zonal asymmetry in Antarctic total ozone during 1979-2014 with respect to the changes in the meteorological variables in the $\mathrm{SH}$ troposphere and lower stratosphere has been presented in the previous section. Regressive, correlative and anomaly composite analyses show that longitudinal shift of the quasi-stationary zonal TOC minimum has a close relationship with changes in the TOC level itself and with the SAM, QSW and SST patterns. Here we discuss our results in the context of the published literature, including analysis of chemistry-climate model attribution simulations. 


\subsection{Relation between the TOC asymmetry and TOC level}

Analysis covers the austral spring months SeptemberNovember and the changes in the seasonally averaged longitude of the $\mathrm{QSW}_{\min }$ characterize the changes in zonal asymmetry of the ozone hole and polar vortex. Zonal asymmetry in the SH stratosphere on monthly and seasonal timescales is formed by the prevailing QSW1 that propagates upward from the troposphere (Sect. 1). In turn, zonal asymmetry in ozone is known to be a factor influencing the planetary wave propagation from the troposphere to the stratosphere (Crook et al., 2008; Albers and Nathan, 2012). Then, the $\mathrm{QSW}_{\min }$ longitude changes could display changes in both the tropospheric wave sources (their activity and distribution) and modification of the stratospheric QSW through the feedback from zonally asymmetric ozone heating.

The results of this work show that, on interannual and decadal timescales, the $\mathrm{QSW}_{\text {min }}$ longitudinal location is in close connection with both the QSW $_{\text {min }}$ level (Fig. 2b and a, respectively) and the overall ozone loss in spring (Fig. 4). Note that ozone mass deficit in Fig. 4 (black curve) characterizes the ozone hole, which is typically asymmetric relative to the South Pole. Therefore, the extent of ozone depletion, which is initially dependent on the wave activity, appears to be largely coupled with the ozone hole asymmetry. For example, in the years of weak (strong) wave activity in spring, large (low) ozone loss is accompanied by eastward (westward) shift of the $\mathrm{QSW}_{\min }$. This is demonstrated by the large simultaneous deviations of the $\mathrm{QSW}_{\min }$ longitude and ozone mass deficit in 1988 and 2002 (Fig. 4), the years when enhanced wave activity resulted in the weakened polar vortices and anomalously small ozone holes (Kodera and Yamazaki, 1989; Allen et al., 2003; Varotsos, 2003b; Grassi et al., 2008; Grytsai et al., 2008).

Generally, planetary wave activity contributes significantly to the interannual variability of the ozone hole size, depth and duration (Kodera and Yamazaki, 1989; Allen et al., 2003; Varotsos, 2002, 2004; Ialongo et al., 2012). However, wave activity did not undergo such significant decadal decrease to cause the decadal tendency in ozone depletion and, therefore, cannot be a contributing factor to the systematic $\mathrm{QSW}_{\text {min }}$ eastward shift. The long-term loss of Antarctic ozone is attributed to the abundance of ODSs peaked near the turn of the century (Salby et al., 2011; Dameris and GodinBeekmann, 2014). Hence, evolution of ODSs is the principal cause of decadal change in the $\mathrm{QSW}_{\min }$ level (Fig. 2a). A related change in asymmetric ozone heating that can potentially affect the planetary wave propagation through the feedback processes (Crook et al., 2008; Albers and Nathan, 2012) could also result in the QSW structure modification. Consistent decadal tendencies seem to support the assumption that evolution of zonally asymmetric ozone depletion (Fig. 2a) could be the main cause of the $\mathrm{QSW}_{\text {min }}$ shift (Fig. 2b). Note that decadal changes of the ozone hole metrics seem to be also influenced by ozone depletion itself. For example, the delay in the final warming of the Antarctic vortex and seasonal disappearance of the ozone hole in the 1980s-1990s appears influenced by increasing overall ozone loss (Haigh and Roscoe, 2009).

Thus, interaction between the planetary waves and ozone could underlay the observed evolution of the asymmetric ozone hole: (i) zonal asymmetry in Antarctic ozone is initially induced by the QSW propagating from the troposphere and (ii) significant change in the TOC level occurs within asymmetric ozone hole, causing change in zonally asymmetric ozone heating that, (iii) through the feedback processes, could result in the QSW structure modification.

\subsection{The SAM pattern}

As is known, positive SAM polarity is associated with enhanced westerlies around Antarctica and decreased surface pressure and air temperature in the polar region (Thompson and Wallace, 2000). The eastward shift of the QSW minimum in total ozone is accompanied by similar indications of the positive SAM polarity: strengthening of zonal wind around Antarctica ( $U-10 \mathrm{~m}$ in Fig. 7d and $U 200$ in Fig. 9f), surface pressure decrease (SP in Fig. 7e) and air temperature decrease ( $T-200$ in Fig. 9e and $T 500$ in Fig. 11) at the SH high latitudes.

Our results do not give information on the direction of the "QSW min $-\mathrm{SAM}$ " coupling. Tropospheric circulation disturbances can influence the evolution of the stratospheric polar vortex, and, in turn, stratospheric processes can induce a tropospheric response that projects on the annular mode (Thompson and Wallace, 2000). It is known that the Antarctic ozone losses in spring impact the SAM-related tropospheric circulation predominantly in summer (Thompson et al., 2011; Schneider et al., 2015), and the spring SAM index shows near-zero decadal trend (Marshall, 2003; Fogt et al., 2009; Arblaster and Gillett, 2014). In addition, in our relationships for zonal wind (Figs. 7a, d, 9b, f), it is difficult to reveal evidence of the poleward shift of the westerly jets observed usually in the positive SAM polarity (Thompson and Wallace, 2000). This is because of strong disturbance of zonal anomaly orientation by the midlatitude QSW3 structure. So, on a decadal timescale, influence of the ozone change accompanied by the $\mathrm{QSW}_{\text {min }}$ longitude change (Fig. 2a and b) on the spring SAM pattern cannot be identified from our results.

Nevertheless, such a feedback possibility cannot be fully excluded, at least on interannual timescales. As shown by Son et al. (2013), stratospheric ozone concentration in September is strongly correlated with the SAM index in October, with $r=-0.7$. The mechanism of this time-lagged downward coupling remains to be determined.

Thus, the appearance of the SAM-like patterns in our relationships (Figs. 6,7 and 9) can be interpreted in two ways that are influenced by interannual variability: first, the 
strength and pattern of the circulation in the spring SH troposphere can conceivably play a significant role in determining the location of the QSW structure in the stratosphere, and, second, the QSW structure in the stratosphere can potentially provide a downward influence on the tropospheric circulation. This could mean that assumed feedback processes of the asymmetric ozone loss (noted in Sect. 4.1 with respect to the QSW structure in the stratosphere) may spread into the troposphere in the springtime. The results by Son et al. (2013) demonstrate such a possibility using an ozone index for the SH polar area. However, ozone variability in the SH polar area is typically combined with ozone asymmetry variability (Fig. 2), and the combined effect of the ozone itself and ozone asymmetry could affect the interannual variability of SH surface climate in spring noted in Son et al. (2013).

\subsection{The QSW1/QSW3 patterns}

Unlike the SAM pattern, the atmospheric QSW3 pattern in the midlatitude troposphere demonstrates long-term changes (Fig. 11) consistent with both the ozone loss tendency (Fig. 2a and 4, black curve) and the $\mathrm{QSW}_{\text {min }}$ longitude shift (Fig. 2b). Positive correlations of up to $r=0.7-0.8$ and the longitudinal shift of the three correlation maxima in Fig. 11 are evidence of significant coupling between $\mathrm{QSW}_{\text {min }}$ and QSW3 on both interannual and decadal timescales. QSW3, although smaller than QSW1, is a dominant feature of the SH midlatitude circulation on daily, seasonal and interannual timescales (Raphael, 2004, and references therein). The QSW3 ridges determined from the $500 \mathrm{hPa}$ geopotential height anomalies over the period 1958-2001 by Raphael (2004) are located climatologically over southern South America, the southern Indian Ocean and southwest of New Zealand.

Those ridge locations correspond to the correlation maxima in Fig. 11a and b for the periods 1979-1992 and 19841997, respectively, which cover the last 2 decades of the time interval in Raphael (2004). The largest eastward shift of the QSW3 pattern occurred between the 1980s and 1990s (Fig. 11a and c, respectively). The central ridge that is located on average near $180^{\circ} \mathrm{E}$ (Fig. 10) drifted from southwest of New Zealand in the 1980s (as in Raphael (2004) and shown in Fig. 11a) to southeast of New Zealand by the early 2000s (Fig. 11d), covering over about $60^{\circ}$ of longitude. Apparent in the longitude sector of this ridge in the 1990s and 2000s has been a cooling of the tropical central Pacific that has been linked to wind-driven vertical circulation changes in the Pacific Ocean (England et al., 2014); this process is also associated with the spin-up of the subtropical gyres and associated surface ocean warming (see Fig. 2 of England et al., 2014) in the region of the ridge. In the 2000s and 2010s, as seen from Fig. 11c-e, the ridge locations showed less drift. In particular, Figure 11e indicates that the central ridge has drifted west back towards New Zealand.
Note that in the years of maximum ozone hole area (easternmost $\mathrm{QSW}_{\min }$ ) the midlatitude wave-3 anomalies of the positive correlation partly cover New Zealand and the southern tip of South America (Fig. 11c-e and Fig. S6c-e in the Supplement). Positive anomaly here corresponds to climate warming in the years of the easternmost $\mathrm{QSW}_{\text {min }}$ migrations. In future, predicted ozone recovery may be accompanied by further westward shift of the wave- 3 pattern and by weakening of the positive anomaly influence in the region of New Zealand and South America (similarly to Fig. 11a and Fig. S6a in the Supplement).

As seen from Fig. 11a and Fig. S6a in the Supplement, both reanalyses show negative correlation anomalies over Australia and East Antarctica in the first time interval 1979-1992 (pre-ozone-hole and first ozone hole years, westernmost $\left.\mathrm{QSW}_{\min }\right)$. Later, these negative anomalies weaken (Fig. 11b-d) and appear again in the latest time interval: 2001-2014 (Fig. 11e). Note that regression in Fig. 6b and correlation in Fig. S2b also show negative anomaly over the southeast part of Australia. These tendencies indicate that Antarctic ozone recovery to pre-ozone-hole levels may be accompanied by strengthening of negative coupling ("tropospheric temperature-QSW ${ }_{\text {min }}$ longitude") in this region. All of these climate effects need further analysis.

The QSW1 structure in the lower stratosphere covers the middle and high SH latitudes and, in the two reanalyses, shows consistent eastward shift between the westernmost and

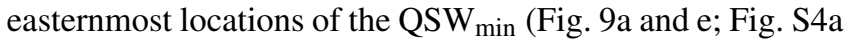
and $\mathrm{S} 4 \mathrm{c}$ in the Supplement). Note that the QSW1 pattern in Fig. 10 takes the altitude range where the vertical ozone profile in the austral spring undergoes the largest ozone decrease (Chubachi, 1984; Varotsos, 2003b; Solomon et al., 2005). Dynamical activity changes the extent of ozone depletion from year to year and, in the conditions of zonal asymmetry of the polar vortex, enhances the TOC variability. The role of zonal asymmetry becomes more important due to vertical non-alignment of the vortex structure (Varotsos, 2004). The vortex appears progressively shifted with height towards South America, and high ozone in the middle stratosphere (around $30 \mathrm{~km}$ ) within the Australian sector masks ozone depletion in the lower stratosphere (Kondragunta et al., 2005; Tully et al., 2008), resulting in additional variability of stratospheric temperature, column ozone and ozone hole characteristics. In this way, vertical changes in the QSW1 structure impact the ozone profile over Antarctica and contribute to the strong correlation between zonal asymmetry in ozone distribution (QSW ${ }_{\min }$ longitude) and stratospheric temperature in Fig. 10.

Overall, the long-term shifts in the QSW3 centers and the QSW1 pattern show a temporal evolution that is qualitatively similar to decadal changes in both ozone depletion (Figs. 2a and 4, black curve) and the shift of QSW $_{\min }$ in total ozone (Fig. 2b). This similarity suggests that decadal-scale changes in the QSW structures in the troposphere and stratosphere could either have an independent common source or result 
from troposphere-stratosphere interaction in the $\mathrm{SH}$ extratropics, including feedback processes assumed in Sects. 4.1 and 4.2. Both possible mechanisms deserve further analysis using observational data and models.

Simulated influence of the greenhouse gases (GHGs) increase on the eastward phase shift in the stratospheric stationary waves (Wang et al., 2013) indicates the possible contribution of the first mechanism. This model simulation links eastward phase shift to the strengthening of the subtropical jet driven by greenhouse gas forcing via sea surface warming. Induced eastward shift is projected by the model to the end of the 21st century (Wang et al., 2013).

Agosta and Canziani (2011) have shown that there are significant interactions/coupling between the ozone layer, the troposphere and the stratosphere during the austral spring, which can be traced by the phase changes in TOC and QSW1 in the stratosphere. Such changes and tropospherestratosphere interactions, by Agosta and Canziani (2011), are linked to both the upward and downward propagation of quasi-stationary wave anomalies. Taking into account the results by Son et al. (2013) and the results of our work, the second of the mentioned mechanisms that suggests contribution of ozone change may be more effective in the recent decades.

\subsection{The SST patterns}

A clear difference between the SST anomaly patterns for the westernmost and easternmost $\mathrm{QSW}_{\min }$ locations has been identified using the two reanalysis data (Fig. 7c and f; Fig. S3c and $f$ in the Supplement). Particularly, the westernmost locations show the negative SST anomaly in the eastern tropical Pacific (La Niña type anomaly; more intense in the NCEP-NCAR reanalysis than in ERA-Interim; Fig. S3c and Fig. 7c). The easternmost locations show the negative SST anomaly in the central tropical Pacific (Fig. 7f and Fig. S3f in the Supplement).

The difference between the two regions of the tropical Pacific in their coupling with the QSW structure in the SH stratosphere was noted by Lin et al. (2012). By Lin et al. (2012), a westward QSW phase shift is seen for negative SST anomalies (La Niña events) in the eastern Pacific, and an eastward shift is seen for warm SST anomalies in the central Pacific. The results of Fig. 7c and Fig. S3c in the Supplement show a similar association between the westernmost QSW $_{\text {min }}$ longitudes and negative SST anomaly in the eastern Pacific. However, the easternmost longitudes in Fig. $7 \mathrm{f}$ and Fig. S3f in the Supplement also show a negative anomaly in the central Pacific, as distinct from a positive one (Lin et al., 2012).

In the individual years, a large westward phase shift in the QSW min occurred in 1988 and 2002 (Figs. 2b and 4). Sources of anomalous planetary waves in these years have been identified in both the tropical Pacific (Kodera and Yamazaki, 1989; Grassi et al., 2008) and the SH extratrop- ics (Nishii and Nakamura, 2004; Peters et al., 2007). It has been noted that the evolutions of sea surface temperatures in the tropical Pacific in the spring months of 1988 and 2002 were different (Varotsos, 2003b), with strong La Niña and emerging El Niño conditions, respectively (McPhaden, 2004; see also time series for the indices Niño 3 and Niño 4 at http://www.esrl.noaa.gov/psd/data/climateindices/).

In the case of 2002 this is counter to the expectation from Lin et al. (2012) based on the prevailing positive central Pacific SST anomaly (McPhaden, 2004) and disagrees with Fig. 7f and Fig. S3f in the Supplement, since a negative SST anomaly exists in this region.

Note that Fig. 7f and Fig. S3f in the Supplement show a significant negative SST anomaly in the South Pacific and positive SST anomalies in the western tropical Pacific and in the Atlantic. Similarly to the PSA mode in the Pacific sector, a poleward-propagating Rossby wave train could be driven by the Atlantic SST anomalies (e.g., Li et al., 2014). Combined influences of the wave train could result in other phase shift direction in the SH stratosphere planetary waves than from positive anomaly in the central tropical Pacific in Lin et al. (2012). In particular, the SH extratropical Rossby wave activity that propagated into the stratosphere in the spring of 2002 (Nishii and Nakamura, 2004; Peters et al., 2007) could also have contributed to the observed QSW $_{\text {min }}$ shift in that year.

In general, the results of Sect. 3 reveal indications of the connection between changes in zonal asymmetry in Antarctic total ozone and changes in zonally symmetric (SAM) and zonally asymmetric (QSW1 and QSW3) patterns in the SH circulation, as well as in the SST patterns. These results are, in general, in agreement with known evidence of coupling between Antarctic ozone and SAM (Thompson and Wallace, 2000; Waugh et al., 2009; Thompson et al., 2011), the SH QSW structure (Agosta and Canziani, 2011; Wang et al., 2013) and the SSTs (Kodera and Yamazaki, 1989; Grassi et al., 2008). These couplings allow us to identify the SH regions where climate changes in spring are accompanied by ozone asymmetry changes.

\subsection{Attribution of longitude shift}

We now turn to chemistry-climate model simulations to further examine long-term changes in the QSW pattern. Here we consider simulations for the Chemistry-Climate Model Initiative (CCMI) (Eyring et al., 2013) produced by the chemistry-climate version of the Australian Community Climate and Earth System Simulator (ACCESS-CCM; Stone, 2015; Stone et al., 2016).

Specifically, we examine a future projection simulation which includes all forcings (REF-C2 scenario) and two sensitivity simulations, one with fixed ODSs (SEN-C2-fODSs scenario) and another with GHGs (SEN-C2-fGHGs scenario), which are described in detail by Stone (2015). The REF-C2 simulation covers the period 1960-2100 and in- 
cludes evolving concentrations of GHGs and ODSs; the SEN-C2-fODS and SEN-C2-fGHG simulations are similar to REF-C2 except that ODSs and GHGs, respectively, are separately fixed at 1960 levels.

The ACCESS-CCM simulations favorably reproduce general characteristics of ozone depletion and stratospheric stationary waves compared with observations and various similar models (Stone, 2015; Stone et al., 2016). In Fig. S7a of the Supplement we show the longitude-height cross section of the correlation between temperature and $\mathrm{QSW}_{\min }$ longitude for the REF-C2 simulation over 1979-2014 for direct comparison with Fig. 10. Although the strength of the correlations in the REF-C2 simulations (Fig. S7a in the Supplement) is weaker than seen in the ERA-Interim reanalysis (Fig. 10) and in the NCEP-NCAR reanalysis (Fig. S5 in the Supplement), the simulation shows similarly located patterns; for example, the region of positive correlation in the lower stratosphere is of similar extent to the reanalyses but shifted $\sim 30^{\circ}$ westward for the REF-C2 simulation. Correlation analysis for an additional model simulation, REFC1, over 1979-2010, which has a similar setup to REF-C2 (Stone, 2015), shows in Fig. S7b in the Supplement a closer agreement with Fig. 10 and Fig. S5 in the Supplement, and we conclude that model internal variability has a noticeable influence on the correlations on these timescales.

Stone (2015) has investigated the long-term shift in the Southern Hemisphere TOC patterns in spring and summer in relation to GHG and ODS changes, where the phase of the wave- 1 component of the TOC (obtained from a zonal Fourier decomposition) was analyzed in a similar manner to Grytsai et al. (2007a). By regressing the wave phase against measures of GHG and ODS forcing, Stone (2015) finds that ODS forcing explains a significant fraction of the long-term variability in the wave-1 longitude compared with GHG in spring at 50 and $60^{\circ} \mathrm{S}$ (his Fig. 5.6), with the strongest influence at the equatorward latitude. From examining also the wave- 1 pattern in temperature at 50 and $500 \mathrm{hPa}$ and zonal wind at $10 \mathrm{hPa}$, Stone (2015) concludes that temperature changes associated with the spring ozone depletion play the most important role in the eastward shift of the TOC pattern at these latitudes, whereas influences from GHG changes are mitigated by a blocking effect from the continental features, particularly the Andes. As noted in Sect. 4.3, simulated GHG increase during the 21 st century shows the eastward phase shift in the stratospheric stationary waves, and this shift is linked to the strengthening of the subtropical jet driven by greenhouse gas forcing via sea surface warming (Wang et al., 2013).

While Stone (2015) only examined the wave-1 phase, we

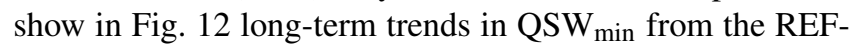
C2, SEN-C2-fODS and SEN-C2-fGHG simulations averaged over 55 to $70^{\circ} \mathrm{S}$, as well as the observational data presented in Fig. 5. While there is large interannual variability, the SEN-C2-fGHG run (which has evolving ozone changes and fixed GHG level) and to a lesser extent the REF-C2 run

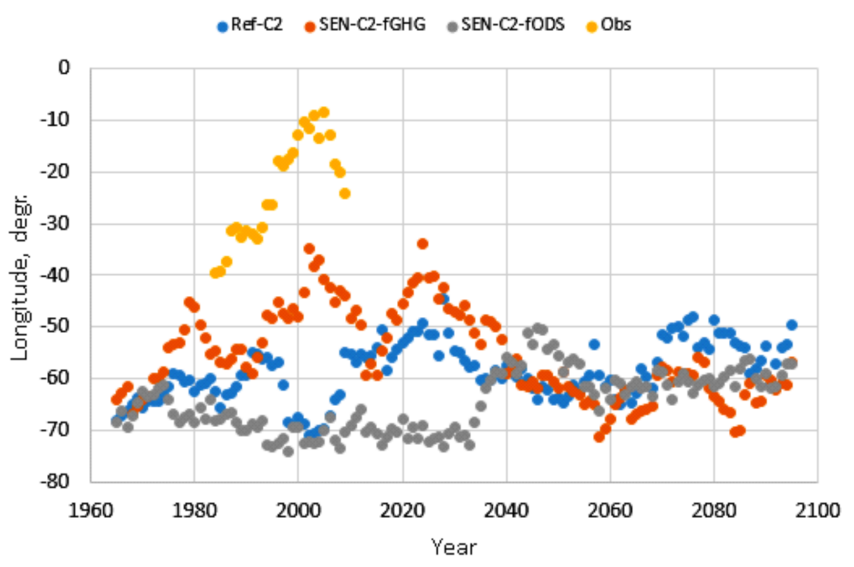

Figure 12. Variation of $\mathrm{QSW}_{\text {min }}$ longitude from the ACCESSCCM model runs (blue: REF-C2; orange: SEN-C2-fGHG; grey: SEN-C2-fODS) compared with the observations (yellow) shown in Fig. 5. For ACCESS-CCM, the $\mathrm{QSW}_{\text {min }}$ longitude was obtained by averaging the results for four latitude bands of $5^{\circ}$ width between 55 and $70^{\circ} \mathrm{S}$ during SON. All time series have been smoothed with an 11 -year running mean (i.e., extending from -5 to +5 years).

(which has both evolving GHG and ODS changes) both show a tendency for an eastward shift (peaking around 2000-2025) and then westward relaxation over the ozone hole period (to about 2060). On the other hand, the SEN-C2-fODS simulation, in which GHGs evolve, shows a much smaller trend and overall less variability over the ozone hole period than the other simulations. We note that, while the observations show a mean phase shifted $\sim 30-40^{\circ}$ further east than the simulations (similar to the difference noted between the positive correlation in the lower stratosphere between Figs. 10 and S7 in the Supplement), the variability in the observational record is not dissimilar to the variability on a similar timescales in the simulations.

Overall we conclude from the simulations that ozone depletion is providing a strong influence on the $\mathrm{QSW}_{\text {min }}$ phase in the current period and may be playing an important role in the level of interannual variability in the stratospheric quasistationary wave pattern, and possibly also the pattern in the troposphere, at least over latitudes around $60^{\circ} \mathrm{S}$.

\section{Conclusions}

We have examined the variability of the minimum in the quasi-stationary pattern in total column ozone in spring at high southern latitudes using observations and model simulations. Our main results are as follows:

1. In interannual variations, the longitude of the QSW minimum in total ozone is in close association with the SAM index, the QSW1/QSW3 patterns in the meteorological variables and the SST patterns. Particularly, the easternmost (westernmost) longitudes of the QSW min- 
imum are accompanied by a shift of the SAM index to positive (negative) polarity (Fig. 8b).

2. On the decadal timescale, consistency between the longitudinal shifts of the QSW minimum in total ozone (Fig. 2b) and the QSW3 pattern in the mid-tropospheric temperature (Fig. 11) has been shown.

3. The SST anomalies over the Pacific and Atlantic basins contribute to the variability in the QSW minimum location.

4. Based on the results from our attribution experiments with the ACCESS-CCM climate model, increased levels of ODSs and GHGs both tend to shift the $\mathrm{QSW}_{\text {min }}$ eastward. From the simulations, asymmetric ozone depletion in spring is likely having a strong influence on the phase of the stratospheric wave pattern around $60^{\circ} \mathrm{S}$.

Regression, correlation, anomaly composite and model analyses show that longitudinal variability at the location of the quasi-stationary zonal TOC minimum has a close relationship with variability in the TOC level itself, in the SAM/QSW patterns in the meteorological variables and in the SST patterns. Therefore, these couplings allow us to identify the $\mathrm{SH}$ regions where the climate variability and climate change in austral spring could be accompanied by the ozone hole asymmetry changes. Related shifts in the tropospheric QSW3 pattern play a role in climate variability in regions of Australia, New Zealand and the southern tip of South America. On the one hand, the results suggest combined influences of the QSW sources on the stationary wave structure in the SH stratosphere; on the other hand, they indicate possible ozone change feedback affecting the wave structure.

Chemistry-climate models predict that the Antarctic ozone will return to the 1980 level in the second half of the 21st century, in the 2050-2070 period (Siddaway et al., 2013; Dameris and Godin-Beekmann, 2014; Solomon et al., 2016), and the period of ozone recovery will take approximately 3 times longer than did the growth epoch of the ozone hole (approximately 2000-2060 and 1980s-1990s, respectively). From our simulations, recovery in ozone levels during the next 2-3 decades will allow the $\mathrm{QSW}_{\text {min }}$ location to generally reverse direction and drift westwards, until the forcing by increasing GHGs starts to dominate and eastward drift resumes (after approximately 2060). The combined impacts of the decadal changes in ozone and GHG on the SH stationary waves indicated by our results suggest that surface influences can be expected over the remainder of this century. It is also possible that interannual variability in the stratospheric stationary waves may reduce as ozone depletion subsides, and this may also have a bearing on interannual variability in high-latitude surface climate. Further model evaluations are needed to assess these indications.

\section{The Supplement related to this article is available online at doi:10.5194/acp-17-1741-2017-supplement.}

Author contributions. Based on ideas developed by Gennadi Milinevsky, Asen Grytsai performed data development and provided analysis with contribution from Gennadi Milinevsky, Andrew Klekociuk, Oleksandr Evtushevsky and Kane Stone. Gennadi Milinevsky, Andrew Klekociuk and Oleksandr Evtushevsky provided additional explanation of the outputs. Asen Grytsai prepared the manuscript with contributions from Gennadi Milinevsky, Andrew Klekociuk and Oleksandr Evtushevsky.

Competing interests. The authors declare that they have no conflict of interest.

Acknowledgements. Authors thank the two anonymous referees for their comments and helpful suggestions. TOMS and OMI daily total ozone data were provided by the Ozone Processing Team, NASA Goddard Space Flight Center, USA, from their Web site at http://ozoneaq.gsfc.nasa.gov. Multi-Sensor Reanalysis data from http://www.temis.nl were used. Data on ozone mass deficit at the southern high latitudes are from http://ozonewatch.gsfc.nasa.gov. NCEP Reanalysis data were provided by the NOAA/OAR/ESRL PSD, Boulder, Colorado, USA, from their Web site at http://www.esrl.noaa.gov/psd/. The ERA-Interim data from the European Centre for Medium-Range Weather Forecasts reanalysis at http://www.ecmwf.int/en/research/climate-reanalysis/era-interim were used. This work was partly supported by Taras Shevchenko National University of Kyiv (project 16BF051-02), the Special Complex Program for Space Research 2012-2016 of the National Academy of Sciences of Ukraine, and the grant F73/115-2016 of the State Fund For Fundamental Research; by the Polar FORCeS project no. 4012 of the Australian Antarctic Science Program, the Australian Research Council's Centre of Excellence for Climate System Science (CE110001028), the Commonwealth Department of the Environment (grant 2011/16853), and the National Institute of Water and Atmospheric Research as part of its New Zealand government-funded core research; and by the Marsden Fund Council from government funding, administered by the Royal Society of New Zealand (grant 12-NIW-006). This research was undertaken with the assistance of resources provided at the $\mathrm{Na}$ tional Computational Infrastructure National Facility systems at the Australian National University through the National Computational Merit Allocation Scheme supported by the Australian Government.

Edited by: J. Kuttippurath

Reviewed by: two anonymous referees

\section{References}

Agosta, E. A. and Canziani, P. O.: Interannual variations in the zonal asymmetry of the subpolar latitudes total ozone column during the austral spring, GeoActa, 35, 1-16, 2010. 
Agosta, E. A. and Canziani, P. O.: Austral spring stratospheric and tropospheric circulation interannual variability, J. Climate, 24, 2629-2647, doi:10.1175/2010JCLI3418.1, 2011.

Albers, J. R. and Nathan, T. N.: Pathways for communicating the effects of stratospheric ozone to the polar vortex: Role of zonally asymmetric ozone, J. Atmos. Sci., 69, 785-801, doi:10.1175/JAS-D-11-0126.1, 2012.

Allen, D. R., Bevilacqua, R. M., Nedoluha, G. E., Randall, C. E., and Manney, G. L.: Unusual stratospheric transport and mixing during the 2002 Antarctic winter, Geophys. Res. Lett., 30, 1599, doi:10.1029/2003GL017117, 2003.

Arblaster, J. M., Gillett, N. P. (Lead Authors), Calvo, N., Forster, P. M., Polvani, L. M., Son, S.-W., Waugh, D. W., and Young, P. J.: Stratospheric ozone changes and climate, Chapter 4 in Scientific Assessment of Ozone Depletion: 2014, Global Ozone Research and Monitoring Project - Report No. 55, World Meteorological Organization, Geneva, Switzerland, 2014.

Baldwin, M., Hirooka, T., O’Neill, A., and Yoden, S.: Major stratospheric warming in the Southern Hemisphere in 2002: Dynamical aspects of the ozone hole split, SPARC Newsletter, 20, 24 27, http://www.sparc-climate.org/publications/newsletter/ (last access: 20 December 2016), 2003.

Brasseur, G. P., Tie, X. X., Rasch, Ph. J., and Lefèvre, F.: A three-dimensional simulation of the Antarctic ozone hole: Impact of anthropogenic chlorine on the lower stratosphere and upper troposphere, J. Geophys. Res., 102, 8909-8930, doi:10.1029/96JD03398, 1997.

Chubachi, S.: Preliminary result of ozone observations at Syowa station from February 1982 to January 1983, Mem. Natl. Inst. Polar Res., 34, 13-19, 1984.

Chubachi, S. and Kajiwara, R.: Total ozone variations at Syowa, Antarctica, Geophys. Res. Lett., 13, 1197-1198, doi:10.1029/GL013i012p01197, 1986.

Crook, J. A., Gillett, N. P., and Keeley, S. P. E.: Sensitivity of Southern Hemisphere climate to zonal asymmetry in ozone, Geophys. Res. Lett., 35, L07806, doi:10.1029/2007GL032698, 2008.

Dameris, M. and Godin-Beekmann, S. (Lead Authors), Alexander, S., Braesicke, P., Chipperfield, M., de Laat, A. T. J., Orsolini, Y., Rex, M., and Santee, M. L.: Update on polar ozone: Past, present, and future, Chapter 3 in Scientific Assessment of Ozone Depletion: 2014, Global Ozone Research and Monitoring Project - Report No. 55, World Meteorological Organization, Geneva, Switzerland, 2014.

Dee, D. P., Uppala, S. M., Simmons, A. J., Berrisford, P., Poli, P., Kobayashi, S., Andrae, U., Balmaseda, M. A., Balsamo, G., Bauer, P., Bechtold, P., Beljaars, A., van de Berg, L., Bidlot, J., Bormann, N., Delsol, C., Dragani, R., Fuentes, M., Geer, A. J., Haimberger, L., Healy, S. B., Hersbach, H., Hólm, E. V., Isaksen, L., Kallberg, P., Köhler, M., Matricardi, M., McNally, A. P., Monge-Sanz, B. M., Morcrette, J.-J., Park, B. K., Peubey, C., de Rosnay, P., Tavolato, C., Thépaut, J.-N., and Vitart, F.: The ERA-Interim reanalysis: configuration and performance of the data assimilation system, Q. J. Roy. Meteor. Soc., 137, 553-597, doi:10.1002/qj.828, data available at: http://www.ecmwf.int/en/ research/climate-reanalysis/era-interim, 2011.

England, M. H., McGregor, S., Spence, P., Meehl, G. A., Timmermann, A., Cai, W., Sen Gupta, A., McPhaden, M. J., Purich, A. and Santoso, A.: Recent intensification of wind-driven circula- tion in the Pacific and the ongoing warming hiatus, Nat. Clim. Change, 4, 222-227, doi:10.1038/nclimate2106, 2014.

Eyring, V., Lamarque, J.-F., Hess, P., Arfeuille, F., Bowman, K., Chipperfield, M. P., Duncan, B., Fiore, A., Gettelman, A., Giorgetta, M. A., Granier, C., Hegglin, M., Kinnison, D., Kunze, M., Langematz, U., Luo, B., Martin, R., Matthes, K., Newman, P. A., Peter, T., Robock, A., Ryerson, T., Saiz-Lopez, A., Salawitch, R., Schultz, M., Shepherd, T. G., Shindell, D., Staehelin, J., Tegtmeier, S., Thomason, L., Tilmes, S., Vernier, J.-P., Waugh, D., and Young, P. Y.: Overview of IGAC/SPARC Chemistry-Climate Model Initiative (CCMI) community simulations in support of upcoming ozone and climate assessments, SPARC Newsletter, 40, 48-66, available at: http://blogs.reading.ac.uk/ccmi/, 2013.

Farman, J. C., Gardiner, B. G., and Shanklin, J. D.: Large losses of total ozone in Antarctica reveal seasonal $\mathrm{ClO}_{x} / \mathrm{NO}_{x}$ interaction, Nature, 315, 207-210, doi:10.1038/315207a0, 1985.

Fogt, R. L., Perlwitz, J., Monaghan, A. J., Bromwich, D. H., Jones, J. M., and Marshall, G. J.: Historical SAM variability. Part II: Twentieth-century variability and trends from reconstructions, observations, and the IPCC AR4 models, J. Climate, 22, 53465365, doi:10.1175/2009JCLI2786.1, 2009.

Fogt, R. L., Jones, J. M., and Renwick, J.: Seasonal zonal asymmetries in the Southern Annular Mode and their impact on regional temperature anomalies, J. Climate, 25, 6253-6270, doi:10.1175/JCLI-D-11-00474.1, 2012a.

Fogt, R. L., Wovrosh, A. J., Langen, R. A., and Simmonds, I.: The characteristic variability and connection to the underlying synoptic activity of the Amundsen-Bellingshausen Seas Low, J. Geophys. Res., 117, D07111, doi:10.1029/2011JD017337, 2012b.

Gabriel, A., Peters, D., Kirchner, I., and Graf, H.-F.: Effect of zonally asymmetric ozone on stratospheric temperature and planetary wave propagation, Geophys. Res. Lett., 34, L06807, doi:10.1029/2006GL028998, 2007.

Gabriel, A., Körnich, H., Lossow, S., Peters, D. H. W., Urban, J., and Murtagh, D.: Zonal asymmetries in middle atmospheric ozone and water vapour derived from Odin satellite data 20012010, Atmos. Chem. Phys., 11, 9865-9885, doi:10.5194/acp-119865-2011, 2011.

Gillett, N. P., Scinocca, J. F., Plummer, D. A., and Reader, M. C.: Sensitivity of climate to dynamically consistent zonal asymmetries in ozone, Geophys. Res. Lett., 36, L10809, doi:10.1029/2009GL037246, 2009.

Grassi, B., Redaelli, G., and Visconti, G.: Tropical SST preconditioning of the SH polar vortex during winter 2002, J. Climate, 21, 5295-5303, doi:10.1175/2008JCLI2136.1, 2008.

Grytsai, A.: Planetary wave peculiarities in Antarctic ozone distribution during 1979-2008, Int. J. Remote Sens., 32, 3139-3152, doi:10.1080/01431161.2010.541518, 2011.

Grytsai, A., Grytsai, Z., Evtushevsky, A., and Milinevsky, G.: Interannual variability of planetary waves in the ozone layer at $65^{\circ} \mathrm{S}$, Int. J. Remote Sens., 26, 3377-3387, doi:10.1080/01431160500076350, 2005.

Grytsai, A. V., Evtushevsky, O. M., Agapitov, O. V., Klekociuk, A. R., and Milinevsky, G. P.: Structure and long-term change in the zonal asymmetry in Antarctic total ozone during spring, Ann. Geophys., 25, 361-374, doi:10.5194/angeo-25-361-2007, 2007a.

Grytsai, A., Evtushevsky, A., Milinevsky, G., and Agapitov, A.: Longitudinal position of the quasi-stationary wave extremes over 
the Antarctic region from the TOMS total ozone, Int. J. Remote Sens., 28, 1391-1396, doi:10.1080/01431160600768021, $2007 \mathrm{~b}$.

Grytsai, A. V., Evtushevsky, O. M., and Milinevsky, G. P.: Anomalous quasi-stationary planetary waves over the Antarctic region in 1988 and 2002, Ann. Geophys., 26, 1101-1108, doi:10.5194/angeo-26-1101-2008, 2008.

Haigh, J. D. and Roscoe, H. K.: The final warming date of the Antarctic polar vortex and influences on its interannual variability, J. Climate, 22, 5809-5819, doi:10.1175/2009JCLI2865.1, 2009.

Hartmann, D. L., Mechoso, C. R., and Yamazaki, K.: Observations of wave-mean flow interaction in the Southern Hemisphere, J. Atmos. Sci., 41, 351-362, doi:10.1175/15200469(1984)041<0351:OOWMFI>2.0.CO;2, 1984.

Hassler, B., Bodeker, G. E., Solomon, S., and Young, P. J.: Changes in the polar vortex: Effects on Antarctic total ozone observations at various stations, Geophys. Res. Lett., 38, L01805, doi:10.1029/2010GL045542, 2011.

Hio, Y. and Hirota, I.: Interannual variations of planetary waves in the Southern Hemisphere stratosphere, J. Meteorol. Soc. Jpn., 80, 1013-1027, 2002.

Hio, Y. and Yoden, S.: Quasi-periodic variations of the polar vortex in the Southern Hemisphere due to wave-wave interaction, J. Atmos. Sci., 61, 2510-2527, doi:10.1175/JAS3257.1, 2004.

Hirota, I. and Hirooka, T.: Normal mode Rossby waves observed in the upper stratosphere. Part I: First symmetric modes of zonal wavenumbers 1 and 2, J. Atmos. Sci., 41, 1253-1267, doi:10.1175/1520-0469(1984)041<1253:NMRWOI>2.0.CO;2, 1984.

Hoppel, K., Bevilacqua, R., Allen, D., Nedoluha, G., and Randall, C.: POAM III observation of the anomalous 2002 Antarctic ozone hole, Geophys. Res. Lett., 30, 1394, doi:10.1029/2003GL016899, 2003.

Huth, R. and Canziani, P. O.: Classification of hemispheric monthly mean stratospheric potential vorticity fields, Ann. Geophys., 21, 805-817, doi:10.5194/angeo-21-805-2003, 2003.

Ialongo, I., Sofieva, V., Kalakoski, N., Tamminen, J., and Kyrölä, E.: Ozone zonal asymmetry and planetary wave characterization during Antarctic spring, Atmos. Chem. Phys., 12, 2603-2614, doi:10.5194/acp-12-2603-2012, 2012.

Kalnay, E., Kanamitsu, M., Kistler, R., Collins, W., Deaven, D., Gandin, L., Iredell, M., Saha, S., White, G., Woollen, J., Zhu, Y., Chelliah, M., Ebisuzaki, W., Higgins, W., Janowiak, J., Mo, K. C., Ropelewski, C., Wang, J., Leetmaa, A., Reynolds, R., Jenne, R., and Joseph, D.: The NCEP/NCAR 40-year Reanalysis Project, B. Am. Meteor. Soc., 77, 1057-1072, doi:10.1175/15200477(1996)077<0437:TNYRP>2.0.CO;2, (data available at: https://www.esrl.noaa.gov/psd/cgi-bin/data/getpage.pl, 1996.

Kodera, K. and Yamazaki, K.: A possible influence of sea surface temperature variation on the recent development of ozone hole, J. Meteorol. Soc. Jpn., 67, 465-472, 1989.

Kondragunta, S., Flynn, L. E., Neuendorffer, A., Miller, A. J., Long, C., Nagatani, R., Zhou, S., Beck, T., Beach, E., McPeters, R., Stolarski, R., Bhartia, P. K., DeLand, M. T., and Huang, L.-K.: Vertical structure of the anomalous 2002 Antarctic ozone hole, J. Atmos. Sci., 52, 801-811, doi:10.1175/JAS-3324.1, 2005.

Kuttippurath, J., Lefèvre, F., Pommereau, J.-P., Roscoe, H. K., Goutail, F., Pazmiño, A., and Shanklin, J. D.: Antarctic ozone loss in 1979-2010: first sign of ozone recovery, Atmos. Chem. Phys., 13, 1625-1635, doi:10.5194/acp-13-1625-2013, 2013.

Li, X., Holland, D. M., Gerber, E. P., and Yoo, C.: Impacts of the north and tropical Atlantic Ocean on the Antarctic Peninsula and sea ice, Nature, 505, 538-542, doi:10.1038/nature12945, 2014.

Lin, P., Fu, Q., Solomon, S., and Wallace, J. M.: Temperature trend patterns in Southern Hemisphere high latitudes: Novel indicators of stratospheric change, J. Climate, 23, 4264-4280, doi:10.1175/2009JCLI2971.1, 2010.

Lin, P., Fu, Q., and Hartmann, D. L.: Impact of tropical SST on stratospheric planetary waves in the Southern Hemisphere, J. Climate, 25. 5030-5046, doi:10.1175/JCLI-D-11-00378.1, 2012.

Malanca, F. E., Canziani, P. O., and Argüello, G. A.: Trends evolution of ozone between 1980 and 2000 at midlatitudes over the Southern Hemisphere: Decadal differences in trends, J. Geophys. Res., 110, D05102, doi:10.1029/2004JD004977, 2005.

Marshall, G. J.: Trends in the Southern Annular Mode from observations and reanalyses, J. Climate, 16, 4134-4143, doi:10.1175/1520-0442(2003)016<4134:TITSAM>2.0.CO;2, 2003.

McPhaden, M. J.: Evolution of the 2002/03 El Niño, B. Am. Meteor. Soc., 85, 677-695, doi:10.1175/BAMS-85-5-677, 2004.

Mo, K. C. and Higgins, R. W.: The Pacific-South American modes and tropical convection during the Southern Hemisphere winter, Mon. Wea. Rev., 126, 1581-1596, doi:10.1175/15200493(1998)126<1581:TPSAMA>2.0.CO;2, 1998.

Newman, P. A. and Nash, E. R.: The unusual Southern Hemisphere stratosphere winter of 2002, J. Atmos. Sci., 62, 614-628, doi:10.1175/JAS-3323.1, 2005.

Newman, P. A., Kawa, S. R., and Nash E. R.: On the size of the Antarctic ozone hole, Geophys. Res. Lett., 31, L21104, doi:10.1029/2004GL020596, 2004.

Nishii, K. and Nakamura, H.: Tropospheric influence on the diminished Antarctic ozone hole in September 2002, Geophys. Res. Lett., 31, L16103, doi:10.1029/2004GL019532, 2004.

Peters, D. and Vargin, P.: Influence of subtropical Rossby wave trains on planetary wave activity over Antarctica in September 2002, Tellus, 67A, 25875, doi:10.3402/tellusa.v67.25875, 2015.

Peters, D., Vargin, P., and Körnich, H.: A study of the zonally asymmetric tropospheric forcing of the austral vortex splitting during September 2002, Tellus, 59A, 384-394, doi:10.1111/j.16000870.2007.00228.x, 2007.

Quintanar, A. I. and Mechoso, C. R.: Quasi-stationary waves in the Southern Hemisphere. Part I: Observational data, J. Climate, 8, 2659-2672, doi:10.1175/15200442(1995)008<2659:QSWITS>2.0.CO;2, 1995.

Randel, W. J.: The seasonal evolution of planetary waves in the southern hemisphere stratosphere and troposphere, Q. J. Roy. Meteor. Soc., 114, 1385-1409, doi:10.1002/qj.49711448403, 1988.

Raphael, M. N.: A zonal wave 3 index for the Southern Hemisphere, Geophys. Res. Lett., 31, L23212, doi:10.1029/2004GL020365, 2004.

Raphael, M. N., Marshall, G. J., Turner, J., Fogt, R. L., Schneider, D., Dixon, D. A., Hosking, J. S., Jones, J. M., and Hobbs, W. R.: The Amundsen Sea Low: Variability, change, and impact on Antarctic climate, B. Am. Meteor. Soc., 97, 111-121, doi:10.1175/BAMS-D-14-00018.1, 2016. 
Roscoe, H. K., Feng, W., Chipperfield, M. P., Trainic, M., and Shuckburgh, E. F.: The existence of the edge region of the Antarctic stratospheric vortex, J. Geophys. Res., 117, D04301, doi:10.1029/2011JD015940, 2012.

Salby, M., Titova, E., and Deschamps, L.: Rebound of Antarctic ozone, Geophys. Res. Lett., 38, L09702, doi:10.1029/2011GL047266, 2011.

Schneider, D. P., Deser, C., and Fan, T.: Comparing the impacts of tropical SST variability and polar stratospheric ozone loss on the Southern Ocean westerly winds, J. Climate, 28, 9350-9372, doi:10.1175/JCLI-D-15-0090.1, 2015.

Siddaway, J. M., Petelina, S. V., Karoly, D. J., Klekociuk, A. R., and Dargaville, R. J.: Evolution of Antarctic ozone in September-December predicted by CCMVal-2 model simulations for the 21st century, Atmos. Chem. Phys., 13, 4413-4427, doi:10.5194/acp-13-4413-2013, 2013.

Solomon, S.: Stratospheric ozone depletion: a review of concepts and history, Rev. Geophys., 37, 275-316, doi:10.1029/1999RG900008, 1999.

Solomon, S., Ivy D. J., Kinnison, D., Mills, M. J., Neely III, R. R., and Schmidt, A.: Emergence of healing in the Antarctic ozone layer, Science, 353, 269-274, doi:10.1126/science.aae0061, 2016.

Solomon, S., Portmann, R. W., Sasaki, T., Hofmann, D. J., and Thompson, D. W. J.: Four decades of ozonesonde measurements over Antarctica, J. Geophys. Res., 110, D21311, doi:10.1029/2005JD005917, 2005.

Son, S.-W., Purich, A., Hendon, H. H., Kim, B.-M., and Polvani, L. M.: Improved seasonal forecast using ozone hole variability?, Geophys. Res. Lett., 40, 6231-6235, doi:10.1002/2013GL057731, 2013.

Stolarski, R. S., Krueger, A. J., Schoeberl M. R., McPeters, R. D., Newman, P. A., and Alpert, J. C.: Nimbus 7 satellite measurements of the springtime Antarctic ozone decrease, Nature, 322 , 808-811, doi:10.1038/322808a0, 1986.

Stone, K. A.: Investigating stratospheric ozone change and associated impacts on circulation and climate, Thesis $(\mathrm{PhD})$, University of Melbourne, available at: http://hdl.handle.net/11343/91558 (last access: 20 December 2016), 2015.

Stone, K. A., Morgenstern, O., Karoly, D. J., Klekociuk, A. R., French, W. J., Abraham, N. L., and Schofield, R.: Evaluation of the ACCESS - chemistry-climate model for the Southern Hemisphere, Atmos. Chem. Phys., 16, 2401-2415, doi:10.5194/acp16-2401-2016, 2016.

Thompson, D. W. J., Solomon, S., Kushner, P. J., England, M. H., Grise, K. M., and Karoly, D. J.: Signatures of the Antarctic ozone hole in Southern Hemisphere surface climate change, Nature Geosci., 4, 741-749, doi:10.1038/NGEO1296, 2011.
Thompson, D. W. J. and Wallace, J.: Annular modes in the extratropical circulation. Part I: Month-to-month variability, J. Climate, 13, 1000-1016, doi:10.1175/15200442(2000)013<1000:AMITEC>2.0.CO;2, 2000.

Tully, M. B., Klekociuk, A. R., Deschamps, L. L., Henderson, S. I., Krummel, P. B., Fraser, P. J., Shanklin, J. D., Downey, A. H., Gies, H. P., and Javorniczky, J.: The 2007 Antarctic ozone hole, Aust. Meteorol. Mag., 57, 279-298, 2008.

Turner, J., Phillips, T., Hosking, J. S., Marshall, G. J., and Orr, A.: The Amundsen Sea low, Int. J. Climatol., 33, 1818-1829, doi:10.1002/joc.3558, 2013.

van $\operatorname{der}$ A, R. J., Allaart, M. A. F., and Eskes, H. J.: Multi sensor reanalysis of total ozone, Atmos. Chem. Phys., 10, 11277 11294, doi:10.5194/acp-10-11277-2010, data available at: http: //www.temis.nl/protocols/O3global.html, 2010.

Varotsos, C.: The Southern Hemisphere ozone hole split in 2002, Environ. Sci. Pollut. Res., 9, 375-376, doi:10.1007/BF02987584, 2002.

Varotsos, C.: What is the lesson from the unprecedented event over Antarctica in 2002? Environ. Sci. Pollut. Res., 10, 80-81, doi:10.1007/BF02980093, 2003a.

Varotsos, C.: Why did a "no-ozone-hole" episode occur in Antarctica?, Eos, 84, 183-185, doi:10.1029/2003EO190007, 2003b.

Varotsos, C.: The extraordinary events of the major, sudden stratospheric warming, the diminutive Antarctic ozone hole, and its split in 2002, Environ. Sci. Pollut. Res., 11, 405-411, doi:doi:10.1007/BF02979661, 2004.

Wang, L., Kushner, P. J. and Waugh, D. W.: Southern Hemisphere stationary wave response to changes of ozone and greenhouse gases, J. Climate, 26, 10205-10217, doi:10.1175/JCLI-D-1300160.1, 2013.

Waugh, D. W. and Randel, W. J.: Climatology of Arctic and Antarctic polar vortices using elliptical diagnostics, J. Atmos. Sci., 56, 1594-1613, doi:10.1175/15200469(1999)056<1594:COAAAP>2.0.CO;2, 1999.

Waugh, D. W., Oman, L., Newman, P. A., Stolarski, R. S., Pawson, S., Nielsen, J. E., and Perlwitz, J.: Effect of zonal asymmetries in stratospheric ozone on simulated Southern Hemisphere climate trends, Geophys. Res. Lett., 36, L18701, doi:10.1029/2009GL040419, 2009.

Wirth, V.: Quasi-stationary planetary waves in total ozone and their correlation with lower stratospheric temperature, J. Geophys. Res., 98, 8873-8882, doi:10.1029/92JD02820, 1993.

WMO (World Meteorological Organization): Scientific Assessment of Ozone Depletion: 2014, Global Ozone Research and Monitoring Project, Report No. 55, 416 pp., Geneva, Switzerland, 2014. 